DOI 10.4171/JEMS/289

Jean Fromentin

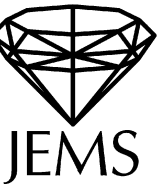

\title{
Every braid admits a short sigma-definite expression
}

Received March 31, 2009

\begin{abstract}
A result by Dehornoy (1992) says that every nontrivial braid admits a $\sigma$-definite expression, defined as a braid word in which the generator $\sigma_{i}$ with maximal index $i$ appears with exponents that are all positive, or all negative. This is the ground result for ordering braids. In this paper, we enhance this result and prove that every braid admits a $\sigma$-definite word expression that, in addition, is quasi-geodesic. This establishes a longstanding conjecture. Our proof uses the dual braid monoid and a new normal form called the rotating normal form.
\end{abstract}

Keywords. Braid group, braid ordering, dual braid monoid, normal form

It has been known since [6] that Artin's braid groups are orderable, by an ordering that enjoys many remarkable properties [11]. The key point in the existence of this ordering is the property that every nontrivial braid admits a $\sigma$-definite expression, defined to be a braid word $w$ in the standard Artin generators $\sigma_{i}$ in which the generator $\sigma_{i}$ with highest index $i$ occurs only positively (no $\sigma_{i}^{-1}$ ), in which case $w$ is called $\sigma$-positive, or only negatively (no $\sigma_{i}$ ), in which case $w$ is called $\sigma$-negative. For $\beta$ a braid, let $\|\beta\|_{\sigma}$ denote the length of the shortest expression of $\beta$ in terms of the Artin generators $\sigma_{i}^{ \pm 1}$. Our main goal in this paper is to prove the following result.

Theorem 1. Each $n$-strand braid $\beta$ admits a $\sigma$-definite expression of length at most $6(n-1)^{2}\|\beta\|_{\sigma}$.

Theorem 1 answers a puzzling open question in the theory of braids. Indeed, the problem of finding a short $\sigma$-definite expression word for every braid has a long history. In the past two decades, at least five or six different proofs of the existence of such $\sigma$ definite expressions have been given. The first one by Dehornoy in 1992 relies on selfdistributive algebra [6]. The next one, by Larue [18], uses the Artin representation of braids as automorphisms of a free group, an argument that was independently rediscovered by Fenn-Greene-Rolfsen-Rourke-Wiest [14] in a topological language of so-called curve diagrams. A completely different proof based on the geometry of the Cayley graph of $B_{n}$ and on Garside's theory appears in [7]. Further methods have been proposed in connection with relaxation algorithms, which are strategies for inductively simplifying

J. Fromentin: Laboratoire de Mathématiques Nicolas Oresme, UMR 6139 CNRS, Université de Caen BP 5186, 14032 Caen, France; e-mail: jean.fromentin@gmail.com

Mathematics Subject Classification (2010): 20F36, 20M05, 06F05 
some geometric object associated with the braid under consideration, typically a family of closed curves drawn in a punctured disk. Both the methods of Dynnikov-Wiest in [12] and of Bressaud in [4] lead to $\sigma$-definite expressions. However, a frustrating feature of all the above methods is that, when one starts with a braid word $w$ of length $\ell$, one obtains in the best case the existence of a $\sigma$-definite word $w^{\prime}$ equivalent to $w$ whose length is bounded above by an exponential in $\ell$-in the cases of $[18,14,7,12,4]$, the original method of [6] is much worse. By contrast, experiments, specially those based on the algorithms derived from [7] and [12], strongly suggested the existence of short $\sigma$-definite expressions, making it natural to conjecture that every braid word of length $\ell$ is equivalent to a $\sigma$-definite word of length $O(\ell)$. This is what Theorem 1 establishes. It is fair to mention that the method of [12] proves the existence of "relatively short $\sigma$-definite expressions". Indeed, it provides for every length $\ell$ braid word a $\sigma$-definite equivalent word whose length with respect to some conveniently extended alphabet lies in $O(\ell)$. However, when the output word is translated back to the alphabet of Artin's generators $\sigma_{i}$, the only upper bound Dynnikov and Wiest could deduce so far is exponential in $\ell$.

The statement of Theorem 1 is essentially optimal. Indeed, it is observed in [11, Chapter XVI] that the length $4(n-2)$ braid word

$$
\sigma_{n-1} \sigma_{n-2}^{-2} \ldots \sigma_{2}^{-2 e} \sigma_{1}^{2 e} \sigma_{2}^{2 e} \ldots \sigma_{n-2}^{2} \sigma_{n-1}^{-1},
$$

with $e= \pm 1$ according to the parity of $n$, is equivalent to no $\sigma$-definite word of length smaller than $n^{2}-n-2$. Thus, in any case, the factor $(n-1)^{2}$ of Theorem 1 could not be possibly replaced with a factor less than $O(n)$.

Our proof of Theorem 1 is effective, and it directly leads to an algorithm that returns, for every $n$-strand braid $\beta$, a distinguished $\sigma$-definite word $\underline{N F}_{n}(\beta)$ that represents $\beta$. Analyzing the complexity of this algorithm leads to

Theorem 2. There exists an effective algorithm which, for each $n$-strand braid specified by a word of length $\ell$, computes the $\sigma$-definite word ${\underline{\mathrm{NF}_{n}}}_{n}(\beta)$ in $O\left(\ell^{2}\right)$ steps.

We prove Theorems 1 and 2 using the dual braid monoid $B_{n}^{+*}$ associated with the BirmanKo-Lee generators and introducing a new normal form on $B_{n}^{+*}$, called the rotating normal form, which is analogous to the alternating normal form of [5] and [10]. The rotating normal form is based on the $\phi_{n}$-splitting operation, a natural way of expressing every $n$-strand dual braid in terms of a finite sequence of $(n-1)$-strand dual braids.

The principle of the argument is as follows. Given an $n$-strand braid $\beta$, we first express it as a fraction $\delta_{n}^{-t} \beta^{\prime}$, where $\delta_{n}$ is the Garside element of the monoid $B_{n}^{+*}$ and $\beta^{\prime}$ belongs to $B_{n}^{+*}$. If the exponent $t$ happens to be greater than the length of the above-mentioned $\phi_{n}$-splitting of $\beta^{\prime}$, then the $\sigma$-negative factor $\delta_{n}^{-t}$ wins over the $\sigma$-positive factor $\beta^{\prime}$, and a $\sigma$-negative word representing $\beta$ can be obtained by an easy direct computation. Otherwise, we determine the rotating normal form $w$ of $\beta^{\prime}$ and try to find a $\sigma$-positive expression of $\beta$ by pushing the negative factor $\delta_{n}^{-t}$ to the right through the positive part $w$. The process is incremental. The problem is that certain special $\sigma$-negative words, called dangerous, appear in the process. The key point is that rotating normal words satisfy some 
syntactic conditions that enable them to neutralize dangerous words. In this way, one finally obtains a word expression of $\beta$ that contains no $\sigma_{n-1}^{-1}$, hence is either $\sigma$-positive, or involves no $\sigma_{n-1}$ at all. An induction on the braid index $n$ then allows one to conclude.

The basic step of the above process consists in switching one dangerous factor and one rotating normal word. This step increases the length by a multiplicative factor 3 at most, and this is the way the length and time upper bounds of Theorems 1 and 2 arise.

In this paper, the braid ordering is not used-on the contrary, the existence of the latter can be (re)-deduced from our current results. However, the braid ordering is present behind our approach. What actually explains the existence of our normal form is the connection between the rotating normal form of Section 2 and the restriction of the braid ordering to the dual braid monoid, which is sketched in [15]. It is also worth noting that the results of the current paper give a new, entirely self-contained proof of the existence of the Dehornoy order of braids.

The paper is organized as follows. In Section 1, we briefly recall the definition of the dual braid monoids and the properties of these monoids that are needed in what follows, in particular those connected with the Garside structure. In Section 2, we introduce the rotating normal form, which is our new normal form on $B_{n}^{+*}$. In Section 3, we establish syntactic constraints on rotating normal words, namely that every normal word is what we call a ladder. In Section 4, we introduce the notion of a dangerous braid word and define the so-called reversing algorithm, which transforms each word consisting of a dangerous word followed by a ladder into a particular type of $\sigma$-definite word called a wall. In Section 5 we compute the complexity of the above reversing algorithm. Finally, we put all pieces together and establish Theorems 1 and 2 in Section 6.

\section{Dual braid monoids}

Our first ingredient for investigating braids will be the Garside structure of the so-called dual braid monoid $B_{n}^{+*}$. Here we recall the needed definitions and results.

\subsection{Birman-Ko-Lee generators}

We recall that Artin's braid group $B_{n}$ is defined for $n \geq 2$ by the presentation

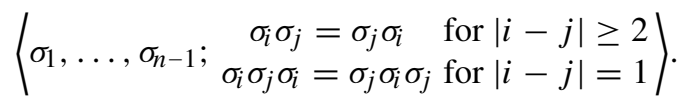

The submonoid of $B_{n}$ generated by $\left\{\sigma_{1}, \ldots, \sigma_{n-1}\right\}$ is denoted by $B_{n}^{+}$, and its elements are called positive braids. As is well known, the monoid $B_{n}^{+}$equipped with Garside's fundamental braid $\Delta_{n}$ has the structure of what is now usually called a Garside monoid $[16,8]$.

The dual braid monoid is another submonoid of $B_{n}$. It is generated by a subset of $B_{n}$ that properly includes $\left\{\sigma_{1}, \ldots, \sigma_{n-1}\right\}$, and consists of the so-called Birman-Ko-Lee generators introduced in [3]. 
Definition 1.1. (See Figure 1.) For $1 \leq p<q$, we put

$$
a_{p, q}=\sigma_{p} \ldots \sigma_{q-2} \sigma_{q-1} \sigma_{q-2}^{-1} \ldots \sigma_{p}^{-1} .
$$
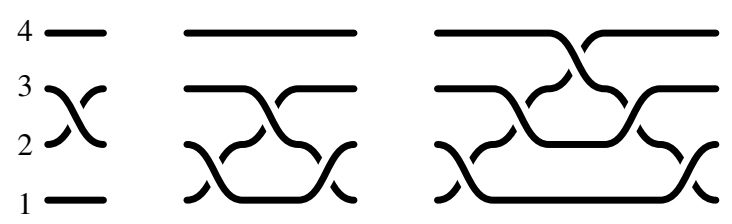

Fig. 1. From left to right: diagram of the braids $a_{2,3}\left(=\sigma_{2}\right), a_{1,3}\left(=\sigma_{1} \sigma_{2} \sigma_{1}^{-1}\right)$ and $a_{1,4}$ $\left(=\sigma_{1} \sigma_{2} \sigma_{3} \sigma_{2}^{-1} \sigma_{1}^{-1}\right)$. The generator $a_{p, q}$ corresponds to the half-twist where the $q$ th strand crosses over the $p$ th strand, both remaining under all intermediate strands.

Remark 1.2. In [3], $a_{p, q}$ is defined to be $\sigma_{q-1} \ldots \sigma_{p+1} \sigma_{p} \sigma_{p+1}^{-1} \ldots \sigma_{q-1}^{-1}$, i.e., it corresponds to the strands at positions $p$ and $q$ passing in front of all intermediate strands, not behind. Both options lead to isomorphic monoids, but our choice naturally leads to the suitable embedding of $B_{n-1}^{+*}$ into $B_{n}^{+*}$, indeed, we need that $B_{n-1}^{+*}$ is an initial segment of $B_{n}^{+*}$ with respect to the standard braid ordering.

The family of all braids $a_{p, q}$ enjoys nice invariance properties with respect to cyclic permutations of indices, which are better visualized when $a_{p, q}$ is represented on a cylinder - see Figure 2. Then it is natural to associate with $a_{p, q}$ the chord connecting the vertices $p$ and $q$ in a circle with $n$ marked vertices [2].
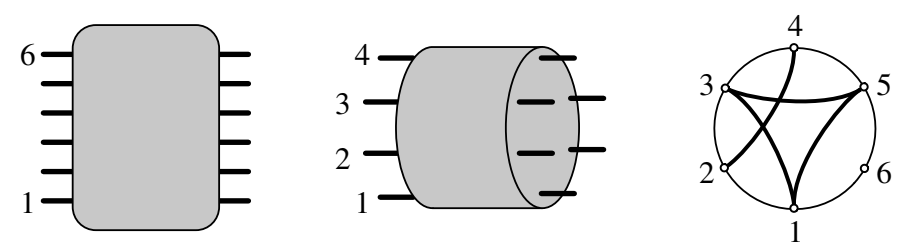

Fig. 2. Rolling up the usual diagram helps to visualize the symmetries of the braids $a_{p, q}$. On the resulting cylinder, $a_{p, q}$ naturally corresponds to the chord connecting the vertices $p$ and $q$.

Hereafter, we write $[p, q]$ for the interval $\{p, \ldots, q\}$ of $\mathbb{N}$, and we say that $[p, q]$ is nested in $[r, s]$ if $r<p<q<s$. A nicely symmetric presentation of $B_{n}$ in terms of the generators $a_{p, q}$ is as follows.

Lemma 1.3 ([3]). In terms of the $a_{p, q}$, the group $B_{n}$ is presented by the relations

$$
\begin{array}{ll}
a_{p, q} a_{r, s}=a_{r, s} a_{p, q} & \text { for }[p, q] \text { and }[r, s] \text { disjoint or nested, } \\
a_{p, q} a_{q, r}=a_{q, r} a_{p, r}=a_{p, r} a_{p, q} & \text { for } 1 \leq p<q<r \leq n .
\end{array}
$$

In the representation of Figure 2, relations of type (1.4) mean that, in each chord triangle, the product of two adjacent edges taken clockwise does not depend on the edges: for instance, the triangle $(1,3,5)$ gives $a_{1,3} a_{3,5}=a_{3,5} a_{1,5}=a_{1,5} a_{1,3}$. Relations of type (1.3) 
say that the generators associated with nonintersecting chords commute: for instance, in Figure 2, we read that $a_{2,4}$ and $a_{1,5}$ commute—but, for instance, nothing is claimed about $a_{2,4}$ and $a_{1,3}$.

\subsection{The dual braid monoid $B_{n}^{+*}$ and its Garside structure}

By definition, we have $\sigma_{p}=a_{p, p+1}$ for each $p$ : every Artin generator is a Birman-KoLee generator. On the other hand, the braid $a_{1,3}$ belongs to no monoid $B_{n}^{+}$. Hence, for $n \geq 3$, the submonoid of $B_{n}$ generated by the Birman-Ko-Lee braids $a_{p, q}$ is a proper extension of $B_{n}^{+}$: this submonoid is what is called the dual braid monoid.

Definition 1.4. For $n \geq 2$, the dual braid monoid $B_{n}^{+*}$ is defined to be the submonoid of $B_{n}$ generated by the braids $a_{p, q}$ with $1 \leq p<q \leq n$.

So, every positive $n$-strand braid belongs to $B_{n}^{+*}$, but the converse is not true for $n \geq 3$ : the braid $a_{1,3}$, i.e., $\sigma_{1} \sigma_{2} \sigma_{1}^{-1}$, belongs to $B_{3}^{+*}$ but not to $B_{3}^{+}$.

Proposition 1.5 ([3]). For each $n$, the relations of Lemma 1.3 yield a presentation of $B_{n}^{+*}$ in terms of the generators $a_{p, q}$, and $B_{n}^{+*}$ is a Garside monoid with Garside element

$$
\delta_{n}=a_{1,2} a_{2,3} \ldots a_{n-1, n} \quad\left(=\sigma_{1} \sigma_{2} \ldots \sigma_{n-1}\right) .
$$

Proposition 1.5 implies that the left- and right-divisibility relations in the dual braid monoid $B_{n}^{+*}$ have lattice properties, i.e., any two elements of $B_{n}^{+*}$ admit (left and right) greatest common divisors and least common multiples. It also implies that $B_{n}$ is a group of fractions for the monoid $B_{n}^{+*}$, and that every element of $B_{n}^{+*}$ admits a distinguished decomposition similar to the greedy normal form of $B_{n}^{+}$[3]. This decomposition involves the so-called simple elements of $B_{n}^{+*}$, which are the divisors of $\delta_{n}$, and are in one-to-one correspondence with the noncrossing partitions of $\{1, \ldots, n\}[3,1]$.

\subsection{The rotating automorphism}

An important role will be played by the so-called rotating automorphism $\phi_{n}$ of $B_{n}^{+*}$. In every Garside monoid, conjugating under the Garside element defines an automorphism [8]. In the case of the monoid $B_{n}^{+}$and its Garside element $\Delta_{n}$, the associated automorphism is the flip automorphism that exchanges $\sigma_{i}$ and $\sigma_{n-i}$ for each $i$, thus an involution that corresponds to a symmetry in braid diagrams. In the case of the dual monoid $B_{n}^{+*}$ and its Garside element $\delta_{n}$, the associated automorphism has order $n$, and it is similar to a rotation.

Lemma 1.6 (See Figure 3.). For each $\beta$ in $B_{n}^{+*}$, let $\phi_{n}(\beta)$ be defined by

$$
\delta_{n} \beta=\phi_{n}(\beta) \delta_{n} \text {. }
$$

Then, for all $p, q$ with $1 \leq p<q \leq n$, we have

$$
\phi_{n}\left(a_{p, q}\right)= \begin{cases}a_{p+1, q+1} & \text { for } q \leq n-1, \\ a_{1, p+1} & \text { for } q=n\end{cases}
$$




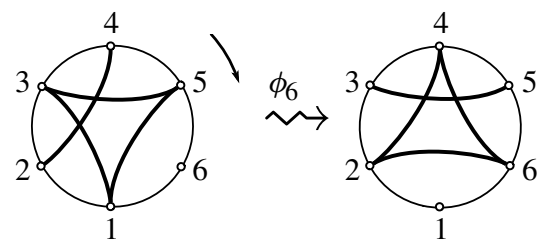

Fig. 3. Representation of the rotating automorphism $\phi_{n}$ as a clockwise rotation of the marked circle by $2 \pi / n$.

The proof is an easy verification from (1.2), (1.5) and the relations (1.3), (1.4). Note that the relation $\phi_{n}\left(a_{p, q}\right)=a_{p+1, q+1}$ always holds provided the indices are taken $\bmod n$ and possibly switched so that, for instance, $a_{p+1, n+1}$ means $a_{1, p+1}$.

The formulas of (1.7) show that $B_{n}^{+*}$ is globally invariant under $\phi_{n}$. By contrast, note that $B_{n}^{+*}$ is not invariant under the flip automorphism $\Phi_{n}: \sigma_{i} \mapsto \sigma_{n-i}$. For instance, $\Phi_{3}\left(a_{1,3}\right)$, which is $\sigma_{2} \sigma_{1} \sigma_{2}^{-1}$, does not belong to $B_{3}^{+*}$.

\section{The rotating normal form}

Besides the Garside structure, the main tool we shall use in this paper is a new normal form for the elements of the dual braid monoid $B_{n}^{+*}$, i.e., a new way of associating with every element of $B_{n}^{+*}$ a distinguished word (in the letters $a_{p, q}$ ) that represents it. This normal form is called the rotating normal form, as it relies on the rotating automorphism $\phi_{n}$ which we have seen is similar to a rotation.

The rotating normal form is reminiscent of the alternating normal form introduced in [10] for the case of the monoid $B_{n}^{+}$-which is itself connected with Burckel's approach of [5]. It is also closely connected with the normal forms introduced in [17], which are other developments, in a different direction, of the alternating normal form. As the properties of $B_{n}^{+*}$ and $\phi_{n}$ are essentially the same as those of $B_{n}^{+}$and $\Phi_{n}$, adapting the results of [10] is easy, and therefore constructing the rotating normal form is not very hardwhat will be harder is identifying the needed properties of rotating normal words, as will be done in subsequent sections.

\subsection{The $\phi_{n}$-splitting}

The basic observation of [10] is that each braid in the monoid $B_{n}^{+}$admits a unique maximal right-divisors that lies in the submonoid $B_{n-1}^{+}$. A similar phenomenon occurs in the dual monoid $B_{n}^{+*}$.

Lemma 2.1. For $n \geq 3$, every braid $\beta$ of $B_{n}^{+*}$ admits a maximal right-divisor lying in $B_{n-1}^{+*}$. The latter is the unique right-divisor $\beta_{1}$ of $\beta$ such that $\beta \beta_{1}^{-1}$ has no nontrivial (i.e., $\neq 1)$ right-divisor lying in $B_{n-1}^{+*}$.

Proof. The submonoid $B_{n-1}^{+*}$ of $B_{n}^{+*}$ is closed under right-divisors and left-lcm's. Hence we can apply Lemma 1.12 of [10]. 
Definition 2.2. The braid $\beta_{1}$ of Lemma 2.1 is called the $B_{n-1}^{+*}$-tail of $\beta$ and it is denoted by $\operatorname{tail}_{n-1}(\beta)$.

Example 2.3. Let us compute the $B_{2}^{+*}$-tail of $\delta_{3}^{2}$. As $B_{2}^{+*}$ is generated by $a_{1,2}$, this $B_{2}^{+*}$-tail is the maximal power of $a_{1,2}$ that right-divides $\delta_{3}^{2}$. By definition, we have $\delta_{3}^{2}=a_{1,2} a_{2,3} a_{1,2} a_{2,3}$. By applying (1.4) twice, we obtain

$$
\delta_{3}^{2}=a_{1,2} a_{2,3} a_{1,3} a_{1,2}=a_{1,2} a_{1,3} a_{1,2}^{2} .
$$

As the word $a_{1,2} a_{1,3}$ is alone in its equivalence class, the braid it represents cannot be right-divisible by $a_{1,2}$. Therefore, the $B_{2}^{+*}$-tail of $\delta_{3}^{2}$ is $a_{1,2}^{2}$.

In the context of the monoid $B_{n}^{+}$, one obtains a distinguished decomposition for every braid in $B_{n}^{+}$by considering the $B_{n-1}^{+}$-tail and the $\Phi_{n}\left(B_{n-1}^{+}\right)$-tail alternately, which is possible because $B_{n}^{+}$is generated by $B_{n-1}^{+}$and $\Phi_{n}\left(B_{n-1}^{+}\right)$. In our context of $B_{n}^{+*}$, we shall use the $B_{n-1}^{+*}$-tail, the $\phi_{n}\left(B_{n-1}^{+*}\right)$-tail, $\ldots$, the $\phi_{n}^{n-1}\left(B_{n-1}^{+*}\right)$-tail cyclically to obtain a distinguished decomposition for every braid of $B_{n}^{+*}$.

In order to show that every braid in $B_{n}^{+*}$ admits such a decomposition, we must check that the images of $B_{n-1}^{+*}$ under the powers of $\phi_{n}$ cover $B_{n}^{+*}$. Actually, iterating twice is enough.

Lemma 2.4. For $n \geq 3$, every generator $a_{p, q}$ of $B_{n}^{+*}$ belongs to $B_{n-1}^{+*} \cup \phi_{n}\left(B_{n-1}^{+*}\right) \cup$ $\phi_{n}^{2}\left(B_{n-1}^{+*}\right)$.

Proof. For $q \leq n-1$, the braid $a_{p, q}$ belongs to $B_{n-1}^{+*}$. Next, for $q=n$ and $p \geq 2$, we have $a_{p, n}=\phi_{n}\left(a_{p-1, n-1}\right)$, which belongs to $\phi_{n}\left(B_{n-1}^{+*}\right)$. Finally, for $p=1$ and $q=n$, we find $a_{p, q}=\phi_{n}\left(a_{n-1, n}\right)=\phi_{n}^{2}\left(a_{n-2, n-1}\right)$, which belongs to $\phi_{n}^{2}\left(B_{n-1}^{+*}\right)$.

By iterating the tail construction, we then associate with every braid of $B_{n}^{+*}$ a finite sequence of braids of $B_{n-1}^{+*}$ that specifies it completely.

Proposition 2.5. Assume $n \geq 3$. Then, for each nontrivial braid $\beta$ of $B_{n}^{+*}$, there exists a unique sequence $\left(\beta_{b}, \ldots, \beta_{1}\right)$ in $B_{n-1}^{+*}$ satisfying $\beta_{b} \neq 1$ and

$$
\beta=\phi_{n}^{b-1}\left(\beta_{b}\right) \cdot \ldots \cdot \phi_{n}\left(\beta_{2}\right) \cdot \beta_{1},
$$

for each $k \geq 1$, the braid $\beta_{k}$ is the $B_{n-1}^{+*}$-tail of $\phi_{n}^{b-k}\left(\beta_{b}\right) \cdot \ldots \cdot \beta_{k}$.

Proof. Starting from $\beta^{(0)}=\beta$, we define two sequences, denoted $\beta^{(k)}$ and $\beta_{k}$, by

$$
\beta^{(k)}=\phi_{n}^{-1}\left(\beta^{(k-1)} \beta_{k}^{-1}\right) \quad \text { and } \quad \beta_{k}=\operatorname{tail}_{n-1}\left(\beta^{(k-1)}\right) \quad \text { for } k \geq 1 .
$$

Using induction on $k \geq 1$, we prove the relations

$$
\begin{aligned}
& \beta=\phi_{n}^{k}\left(\beta^{(k)}\right) \cdot \phi_{n}^{k-1}\left(\beta_{k}\right) \cdot \ldots \cdot \beta_{1}, \\
& \operatorname{tail}_{n-1}\left(\phi_{n}\left(\beta^{(k)}\right)\right)=1 .
\end{aligned}
$$


Assume $k=1$. Lemma 2.1 implies that the $B_{n-1}^{+*}$-tail of $\beta \beta_{1}^{-1}$ is trivial. Then, as $\phi_{n}\left(\beta^{(1)}\right)$ is equal to $\beta \beta_{1}^{-1}$, the $B_{n-1}^{+*}$-tail of $\phi_{n}\left(\beta^{(1)}\right)$ is trivial, and the relation $\beta=\phi_{n}\left(\beta^{(1)}\right) \cdot \beta_{1}$ holds. Assume $k \geq 2$. By the construction of $\beta^{(k)}$, we have $\phi_{n}\left(\beta^{(k)}\right)=\beta^{(k-1)} \beta_{k}^{-1}$, hence $\beta^{(k-1)}=\phi_{n}\left(\beta^{(k)}\right) \cdot \beta_{k}$. Then we have the relation

$$
\phi_{n}^{k-1}\left(\beta^{(k-1)}\right)=\phi_{n}^{k}\left(\beta^{(k)}\right) \cdot \phi_{n}^{k-1}\left(\beta_{k}\right) .
$$

On the other hand, by induction hypothesis, we have

$$
\beta=\phi_{n}^{k-1}\left(\beta^{(k-1)}\right) \cdot \phi_{n}^{k-2}\left(\beta_{k-1}\right) \cdot \ldots \cdot \beta_{1} .
$$

Substituting (2.6) in (2.7), we obtain (2.4). As $\beta_{k}$ is the $B_{n-1}^{+*}$-tail of $\beta^{(k-1)}$, Lemma 2.1 gives (2.5).

By construction, the sequence of right-divisors of $\beta$,

$$
\beta_{1}, \phi_{n}\left(\beta_{2}\right) \beta_{1}, \phi_{n}^{2}\left(\beta_{3}\right) \phi_{n}\left(\beta_{2}\right) \beta_{1}, \ldots
$$

is nondecreasing for divisibility, and therefore, for length reasons, it must be eventually constant. Hence, by right cancellativity of $B_{n}^{+*}$, there exists $b$ such that for $k \geq b$, we have $\phi_{n}^{k-1}\left(\beta_{k}\right) \cdot \ldots \cdot \beta_{1}=\phi_{n}^{b-1}\left(\beta_{b}\right) \cdot \ldots \cdot \beta_{1}$. Then (2.4) implies

$$
\beta=\phi_{n}^{b}\left(\beta^{(b)}\right) \phi_{n}^{b-1}\left(\beta_{b}\right) \cdot \ldots \cdot \beta_{1},
$$

with $\beta_{b} \neq 1$ whenever $b$ is chosen to be minimal.

By the definition of $b$, we have $\beta_{k}=1$, and therefore $\phi_{n}\left(\beta^{(k)}\right)=\beta^{(k-1)}$ by (2.3), for $k \geq b+1$. Then we have $\beta^{(b)}=\phi_{n}\left(\beta^{(b+1)}\right), \phi_{n}^{-1}\left(\beta^{(b)}\right)=\phi_{n}\left(\beta^{(b+2)}\right)$ and $\phi_{n}^{-2}\left(\beta^{(b)}\right)=$ $\phi_{n}\left(\beta^{(b+3)}\right)$. By (2.5), the $B_{n-1}^{+*}$-tails of $\beta^{(b)}, \phi_{n}^{-1}\left(\beta^{(b)}\right)$ and $\phi_{n}^{-2}\left(\beta^{(b)}\right)$ are trivial. Hence, for every generator $x$ of $B_{n-1}^{+*}$, the braid $\beta^{(b)}$ is not right-divisible by $x$, nor by $\phi_{n}(x)$ or $\phi_{n}^{2}(x)$. Then Lemma 2.4 implies that $\beta^{(b)}$ is right-divisible by no $a_{p, q}$ with $1 \leq p<q \leq n$, i.e., we have $\beta^{(b)}=1$, whence $\beta=\phi_{n}^{b-1}\left(\beta_{b}\right) \cdot \ldots \cdot \beta_{1}$.

We now prove the uniqueness of $\left(\beta_{b}, \ldots, \beta_{1}\right)$. Let $\phi_{n}^{c-1}\left(\gamma_{c}\right) \cdot \ldots \cdot \phi_{n}\left(\gamma_{2}\right) \cdot \phi_{n}\left(\gamma_{1}\right)$ be a decomposition of $\beta$ satisfying $\gamma_{c} \neq 1$ and $\gamma_{k}=\operatorname{tail}_{n-1}\left(\phi_{n}^{c-k}\left(\gamma_{c}\right) \cdot \ldots \cdot \gamma_{k}\right)$ for each $k \geq 1$. Using induction on $k \geq 1$, we prove the relations

$$
\gamma_{k}=\beta_{k} \quad \text { and } \quad \phi_{n}^{c-k-1}\left(\gamma_{c}\right) \cdot \ldots \cdot \phi_{n}\left(\gamma_{k+2}\right) \cdot \gamma_{k+1}=\beta^{(k)} .
$$

For $k=1$, by hypothesis, we have $\beta=\left(\phi_{n}^{c-1}\left(\gamma_{c}\right) \cdot \ldots \cdot \phi_{n}\left(\gamma_{2}\right)\right) \cdot \gamma_{1}$, where $\gamma_{1}$ is the $B_{n-1}^{+*}$ tail of $\beta$, hence, by Lemma 2.1 , we have $\beta_{1}=\gamma_{1}$ and $\beta^{(1)}=\phi_{n}^{c-2}\left(\gamma_{c}\right) \cdot \ldots \cdot \phi_{n}\left(\gamma_{3}\right) \cdot \gamma_{2}$. By the induction hypothesis,

$$
\left(\phi_{n}^{c-k-1}\left(\gamma_{c}\right) \cdot \ldots \cdot \phi_{n}\left(\gamma_{k+2}\right)\right) \cdot \gamma_{k+1}=\beta^{(k)},
$$

and by the hypothesis about $\gamma_{k+1}$, the braid $\gamma_{k+1}$ is the $B_{n-1}^{+*}$-tail of $\beta^{(k)}$. Then, by Lemma 2.1 again, $\gamma_{k+1}=\beta_{k+1}$ and $\phi_{n}^{c-k-2}\left(\gamma_{c}\right) \cdot \ldots \cdot \phi_{n}\left(\gamma_{k+3}\right) \cdot \gamma_{k+2}=\beta^{(k+1)}$. We proved $\gamma_{k}=\beta_{k}$ for $b \geq k \geq 1$, hence

$$
\phi_{n}^{c-b-1}\left(\gamma_{c}\right) \cdot \ldots \cdot \phi_{n}\left(\gamma_{b+2}\right) \cdot \gamma_{b+1}=\beta^{(b)}
$$


By the definition of $b$, we have $\beta^{(b)}=1$, whereas, by hypothesis, the braid $\gamma_{c}$ is nontrivial. So (2.8) may hold only for $c=b$.

Definition 2.6. The sequence $\left(\beta_{b}, \ldots, \beta_{1}\right)$ of Proposition 2.5 is called the $\phi_{n}$-splitting of $\beta$. Its length, i.e., the parameter $b$, is called the $n$-breadth of $\beta$.

The idea of the $\phi_{n}$-splitting is very simple: starting with a braid $\beta$ of $B_{n}^{+*}$, we extract the maximal right-divisor that lies in $B_{n-1}^{+*}$, i.e., that leaves the $n$th strand unbraided, then we extract the maximal right-divisor of the remainder that leaves the first strand unbraided, and so on rotating by $2 \pi / n$ at each step—see Figure 4.

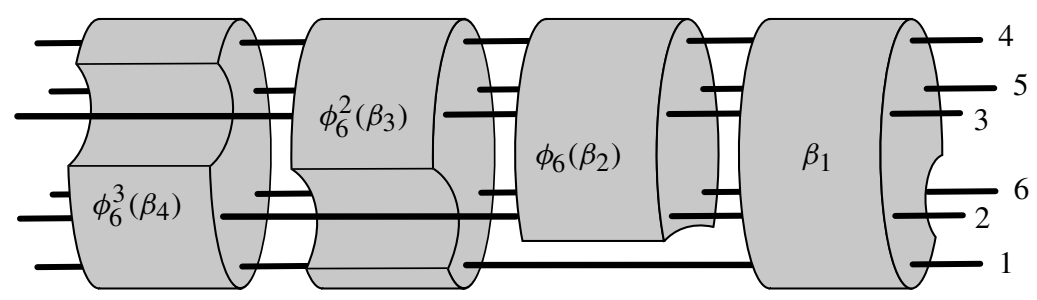

Fig. 4. The $\phi_{6}$-splitting of a braid of $B_{6}^{+*}$. Starting from the right, we extract the maximal rightdivisor that keeps the sixth strand unbraided, then rotate by $2 \pi / 6$ and extract the maximal rightdivisor that keeps the first strand unbraided, etc.

In practice, we shall use the following criterion for recognizing a $\phi_{n}$-splitting.

\section{Lemma 2.7. Condition (2.2) is equivalent to}

$$
\text { for each } k \geq 1 \text {, the } B_{n-1}^{+*} \text {-tail of } \phi_{n}^{b-k}\left(\beta_{b}\right) \cdot \ldots \cdot \phi_{n}\left(\beta_{k+1}\right) \text { is trivial. }
$$

Proof. By Lemma 2.1, for every $k \geq 1$, the braid $\beta_{k}$ is the $B_{n-1}^{+*}$-tail of the braid $\phi_{n}^{b-k}\left(\beta_{b}\right)$. $\ldots \cdot \phi_{n}\left(\beta_{k+1}\right) \cdot \beta_{k}$ if and only if the $B_{n-1}^{+*}$-tail of $\phi_{n}^{b-k}\left(\beta_{b}\right) \cdot \ldots \cdot \phi_{n}\left(\beta_{k+1}\right)$ is trivial. Hence (2.2) and (2.9) are equivalent.

As the notion of $\phi_{n}$-splitting is both new and fundamental for what follows, we mention several examples.

Example 2.8. Let us first determine the $\phi_{n}$-splitting of the Birman-Ko-Lee generators of $B_{n}^{+*}$. For $q \leq n-1$, the braid $a_{p, q}$ belongs to $B_{n-1}^{+*}$, so its $\phi_{n}$-splitting is $\left(a_{p, q}\right)$. As $a_{p, n}$ does not lie in $B_{n-1}^{+*}$, the rightmost entry in its $\phi_{n}$-splitting must be 1 . Now, we have $\phi_{n}^{-1}\left(a_{p, n}\right)=a_{p-1, n-1}$ for $p \geq 2$. Hence, for $p \geq 2$, the $\phi_{n}$-splitting of $a_{p, n}$ is $\left(a_{p-1, n-1}, 1\right)$. Finally, the braids $a_{1, n}$ and $\phi_{n}^{-1}\left(a_{1, n}\right)=a_{n-1, n}$ do not lie in $B_{n-1}^{+*}$, but $\phi_{n}^{-2}\left(a_{1, n}\right)=a_{n-2, n-1}$ does. So the $\phi_{n}$-splitting of $a_{1, n}$ is the sequence $\left(a_{n-2, n-1}, 1,1\right)$. To summarize, the $\phi_{n}$-splitting of $a_{p, q}$ is

$$
\begin{cases}\left(a_{p, q}\right) & \text { for } p<q \leq n-1, \\ \left(a_{p-1, n-1}, 1\right) & \text { for } 2 \leq p \text { and } q=n \\ \left(a_{n-2, n-1}, 1,1\right) & \text { for } p=1 \text { and } q=n .\end{cases}
$$


Example 2.9. Let us compute the $\phi_{3}$-splitting of $\delta_{3}^{2}$. With the notation of the proof of Proposition 2.5, we obtain

$$
\begin{array}{ll}
\beta^{(0)}=\beta=\left(a_{1,2} a_{2,3}\right)^{2}, & \beta_{1}=\operatorname{tail}_{2}\left(\beta^{(0)}\right)=a_{1,2}^{2}, \\
\beta^{(1)}=\phi_{3}^{-1}\left(\beta^{(0)} \beta_{1}^{-1}\right)=\phi_{3}^{-1}\left(a_{1,2} a_{1,3}\right)=a_{1,3} a_{2,3}, & \beta_{2}=\operatorname{tail}_{2}\left(\beta^{(1)}\right)=1, \\
\beta^{(2)}=\phi_{3}^{-1}\left(\beta^{(1)} \beta_{2}^{-1}\right)=\phi_{3}^{-1}\left(a_{1,3} a_{2,3}\right)=a_{2,3} a_{1,2}, & \beta_{3}=\operatorname{tail}_{2}\left(\beta^{(2)}\right)=a_{1,2}, \\
\beta^{(3)}=\phi_{3}^{-1}\left(\beta^{(2)} \beta_{3}^{-1}\right)=\phi_{3}^{-1}\left(a_{2,3}\right)=a_{1,2}, & \beta_{4}=\operatorname{tail}_{2}\left(\beta^{(3)}\right)=a_{1,2}, \\
\beta^{(4)}=\phi_{3}^{-1}\left(\beta^{(3)} \beta_{4}^{-1}\right)=1, &
\end{array}
$$

and we stop as the remainder $\beta^{(4)}$ is trivial. Thus the $\phi_{3}$-splitting of $\delta_{3}^{2}$ is the sequence $\left(a_{1,2}, a_{1,2}, 1, a_{1,2}^{2}\right)$.

\subsection{The rotating normal form}

Using the $\phi_{n}$-splitting, we shall now construct a unique normal form for the elements of $B_{n}^{+*}$, i.e., we identify for each braid $\beta$ in $B_{n}^{+*}$ a distinguished word that represents $\beta$.

The principle is as follows. First, each braid of $B_{2}^{+*}$ is represented by a unique word $a_{1,2}^{k}$. Then the $\phi_{n}$-splitting provides a distinguished decomposition for every braid of $B_{n}^{+*}$ in terms of braids of $B_{n-1}^{+*}$. So, using induction on $n$, we can define a normal form for $\beta$ in $B_{n}^{+*}$ starting with the normal form of the entries in the $\phi_{n}$-splitting of $\beta$.

For the rest of this paper, it will be convenient to make the following conventions for braid words and the braids they represent.

Definition 2.10. A word on the letters $\sigma_{i}^{ \pm 1}$ (resp. $a_{p, q}^{ \pm 1}$ ) is called a $\sigma$-word (resp. an $a$ word). The set of all positive $n$-strand $a$-words (including the empty word $\varepsilon$ ) is denoted by $\underline{B}_{n}^{+*}$. The braid represented by an $a$-word or a $\sigma$-word $w$ is denoted by $\bar{w}$. For $w$ a $\sigma$-word or an $a$-word and $w^{\prime}$ a $\sigma$-word or an $a$-word, we say that $w$ is equivalent to $w^{\prime}$, denoted $w \equiv w^{\prime}$, if $\bar{w}=\overline{w^{\prime}}$.

According to the formulas (1.7), $\phi_{n}$ maps each braid $a_{p, q}$ to another similar braid $a_{r, s}$. Using this observation, we can introduce the alphabetical homomorphism, still denoted $\phi_{n}$, that maps the letter $a_{p, q}$ to the corresponding letter $a_{r, s}$, and extends to every $a$-word. Note that, in this way, if the $a$-word $w$ represents the braid $\beta$, then $\phi_{n}(w)$ represents $\phi_{n}(\beta)$.

Definition 2.11. (i) For $\beta$ in $B_{2}^{+*}$, the $\phi_{2}$-rotating normal form of $\beta$ is defined to be the unique $a$-word $a_{1,2}^{k}$ that represents $\beta$.

(ii) For $\beta$ in $B_{n}^{+*}$ with $n \geq 3$, the $\phi_{n}$-rotating normal form of $\beta$ is defined to be the $a$-word $\phi_{n}^{b-1}\left(w_{b}\right) \ldots w_{1}$, where $\left(\beta_{b}, \ldots, \beta_{1}\right)$ is the $\phi_{n}$-splitting of $\beta$ and $w_{k}$ is the $\phi_{n-1^{-}}$ rotating normal form of $\beta_{k}$ for each $k$.

As the $\phi_{n}$-splitting of a braid $\beta$ lying in $B_{n-1}^{+*}$ is the length 1 sequence $(\beta)$, the $\phi_{n}$ normal form and the $\phi_{n-1}$-normal form of $\beta$ coincide. Therefore, we can drop the subscript $n$, and speak of the rotating normal form, or simply normal form, of a braid of $B_{n}^{+*}$. 
We naturally say that a positive $a$-word is normal if it is the normal form of the braid it represents.

Example 2.12. Let us compute the normal form of $\delta_{4}^{2}$. First, we check the equality $\delta_{4}^{2}=$ $a_{1,2} a_{1,4} \delta_{3}^{2}$. Then the $\phi_{4}$-splitting of $\delta_{4}^{2}$ turns out to be $\left(a_{2,3}, a_{2,3}, 1, \delta_{3}^{2}\right)$. The $\phi_{3}$-splitting of $a_{2,3}$ is $\left(a_{1,2}, 1\right)$, and therefore its normal form is $\phi_{3}\left(a_{1,2}\right)$, which is $a_{2,3}$. Next, we saw in Example 2.9 that the $\phi_{3}$-splitting of $\delta_{3}^{2}$ is $\left(a_{1,2}, a_{1,2}, 1, a_{1,2}^{2}\right)$. Therefore, its normal form is $\phi_{3}^{3}\left(a_{1,2}\right) \cdot \phi_{3}^{2}\left(a_{1,2}\right) \cdot \phi_{3}(\varepsilon) \cdot a_{1,2} a_{1,2}$, hence $a_{1,2} \cdot a_{1,3} \cdot \varepsilon \cdot a_{1,2} a_{1,2}$, i.e., $a_{1,2} a_{1,3} a_{1,2} a_{1,2}$. So, finally, the normal form of $\delta_{4}^{2}$ is

$$
\phi_{4}^{3}\left(a_{2,3}\right) \cdot \phi_{4}^{2}\left(a_{2,3}\right) \cdot \phi_{4}(\varepsilon) \cdot a_{1,2} a_{1,3} a_{1,2} a_{1,2},
$$

hence $a_{1,2} \cdot a_{1,4} \cdot \varepsilon \cdot a_{1,2} a_{1,3} a_{1,2} a_{1,2}$, i.e., $a_{1,2} a_{1,4} a_{1,2} a_{1,3} a_{1,2} a_{1,2}$. When expressed in terms of the standard Artin generators, this amounts to saying that the rotating normal form of the braid $\delta_{4}^{2}$, i.e., $\left(\sigma_{1} \sigma_{2} \sigma_{3}\right)^{2}$, is $\sigma_{1} \cdot \sigma_{1} \sigma_{2} \sigma_{3} \sigma_{2}^{-1} \sigma_{1}^{-1} \cdot \sigma_{1} \cdot \sigma_{1} \sigma_{2} \sigma_{1}^{-1} \cdot \sigma_{1} \cdot \sigma_{1}$, a result that has no interest in itself but to show how different the current normal form is from the usual normal forms.

As the relations of Lemma 1.3 preserve the length, positive equivalent $a$-words always have the same length. Hence, if $w^{\prime}$ is the unique normal word equivalent to some word $w$ of $\underline{B}_{n}^{+*}$, then $w$ and $w^{\prime}$ have the same length.

Proposition 2.13. For each length $\ell$ word $w$ of $\underline{B}_{n}^{+*}$, the normal form of $\bar{w}$ can be computed in at most $O\left(\ell^{2}\right)$ elementary steps.

Proof. Computing the $B_{n-1}^{+*}$-tail of the braid $\bar{w}$ can be done in $O(\ell)$ steps. Hence computing the $\phi_{n}$-splitting can be done in $O\left(\ell^{2}\right)$ steps. Taking into account the observation that the lengths of equivalent words are equal, one deduces using an easy induction on $n$ that computing the rotating normal form of $\bar{w}$ can be done in $O\left(\ell^{2}\right)$ steps.

We considered above the question of going from $w$ to an equivalent normal word, thus first identifying the $\phi_{n}$-splitting of $w$ and then finding the normal form of the successive entries. Conversely, when we start with a normal word $w$, it is easy to isolate the successive entries of the $\phi_{n}$-splitting of the braid $\bar{w}$, i.e., to group the successive letters in blocks: one finds the last block as the maximal block that contains no letter $a_{i, n}$, then one removes this block, applies the rotation $\phi_{n}^{-1}$, and iterates the process.

Hereafter, if $w$ is an $n$-normal word, the (unique) sequence of $(n-1)$-normal words of $\left(w_{b}, \ldots, w_{1}\right)$ such that $\left(\overline{w_{b}}, \ldots, \overline{w_{1}}\right)$ is the $\phi_{n}$-splitting of $\bar{w}$ is naturally called the $\phi_{n}$-splitting of $w$.

Lemma 2.14. Assume $n \geq 3$. For each normal word $w$ of $\underline{B}_{n}^{+*}$, the $\phi_{n}$-splitting of $w$ can be computed in at most $O(\ell)$ elementary steps.

Proof. By the definition of $\phi_{n}$, a generator $a_{p, q}$ lies in $\phi_{n}^{k}\left(B_{n-1}^{+*}\right)$ if and only if we have $p \neq k \bmod n$ and $q \neq k \bmod n$. Therefore, given a normal word $w$ in $\underline{B}_{n}^{+*}$, we can directly read the $\phi_{n}$-splitting $\left(w_{b}, \ldots, w_{1}\right)$ of $w$. Indeed, reading $w$ from right to left, $w_{1}$ is the maximal suffix of $w$ that lies in $\underline{B}_{n-1}^{+*}$, then $\phi_{n}\left(w_{2}\right)$ is the maximal suffix of the remaining braid lying in $\phi_{n}\left(B_{n-1}^{+*}\right)$, etc., until the empty word is left. 
Example 2.15. Let us consider the normal word $w=a_{1,2} a_{1,4} a_{2,3} a_{1,2}$ and compute the $\phi_{4}$-splitting of $w$. Reading $w$ from right to left, we find that the maximal suffix of $w$ containing no letter $a_{p, q}$ with $p=0 \bmod n$ or $q=0 \bmod n$ is the word $a_{2,3} a_{1,2}$. The latter is the maximal suffix of $w$ lying in $\underline{B}_{3}^{+*}$, so we have the relation $w_{1}=a_{2,3} a_{1,2}$. Repeating this process, one easily finds that the $\phi_{4}$-splitting of $w$ is $\left(\phi_{4}^{-3}\left(a_{1,2}\right), \phi_{4}^{-2}\left(a_{1,4}\right), \phi_{4}^{-1}(1), a_{2,3} a_{1,2}\right)$, hence the sequence $\left(a_{2,3}, a_{2,3}, 1, a_{2,3} a_{1,2}\right)$.

\section{Ladders}

The $\phi_{n}$-splitting operation associates with every braid in $B_{n}^{+*}$ a finite sequence of braids in $B_{n-1}^{+*}$. Now, in the other direction, a sequence of braids in $B_{n-1}^{+*}$ need not be the $\phi_{n}$ splitting of a braid in $B_{n}^{+*}$. The aim of this section is to establish constraints that are satisfied by the entries of a $\phi_{n}$-splitting. The main constraint is that a $\phi_{n}$-splitting necessarily contains what we call ladders, which are sequences of (nonadjacent) letters $a_{p, q}$ whose indices $q$ make an increasing sequence (the bars of the ladder).

\subsection{Last letters}

We begin with some elementary observations about the last letters of the normal forms of the entries in a $\phi_{n}$-splitting.

Definition 3.1. For each nonempty word $w$, the last letter of $w$ is denoted by $w^{\#}$. Then, for each nontrivial braid $\beta$ in $B_{n}^{+*}$, we define the last letter of $\beta$, denoted $\beta^{\#}$, to be the last letter in the normal form of $\beta$.

Lemma 3.2. Assume $n \geq 3$, and let $\left(\beta_{b}, \ldots, \beta_{1}\right)$ be a $\phi_{n}$-splitting.

(i) For $k \geq 2$, the letter $\beta_{k}^{\#}$ is $a_{p, n-1}$ for some $p$, unless $\beta_{k}=1$.

(ii) For $k \geq 3$, we have $\beta_{k} \neq 1$.

(iii) For $k \geq 2$, if the normal form of $\beta_{k}$ is $w a_{n-2, n-1}$ with $w$ nonempty, then the letter $w^{\#}$ is $a_{p, n-1}$ for some $p$.

Proof. (i) Assume $k \geq 2$. Put $a_{p, q}=\beta_{k}^{\#}$. By (2.9), the $B_{n-1}^{+*}$-tail of $\phi_{n}^{b-k+1}\left(\beta_{b}\right) \cdot \ldots \cdot \phi_{n}\left(\beta_{k}\right)$ is trivial. In particular, $\phi_{n}\left(\beta_{k}^{\#}\right)$ cannot lie in $B_{n-1}^{+*}$, so $\beta_{k}^{\#}$ must be a letter of the form $a_{p, n-1}$.

(ii) Assume that $\beta_{c}=1$ with $c \geq 3$ and $\beta_{k} \neq 1$ for $b \geq k>c$. By the definition of a $\phi_{n}$-splitting, we have $\beta_{b} \neq 1$, hence $c \leq b-1$. By the definition of $c$, we have $\beta_{c+1} \neq 1$, hence, by (i), $\beta_{c+1}^{\#}=a_{r, n-1}$ for some $r$. By (2.9), the $B_{n-1}^{+*}$-tail of $\phi_{n}^{b-c+1}\left(\beta_{b}\right) \cdot \ldots$. $\phi_{n}^{2}\left(\beta_{c+1}\right) \phi_{n}\left(\beta_{c}\right)$ is 1 . As $\beta_{c}=1$, we deduce that the $B_{n-1}^{+*}$-tail of $\phi_{n}^{b-c+1}\left(\beta_{b}\right) \cdot \ldots \cdot \phi_{n}^{2}\left(\beta_{c+1}\right)$ is 1 as well. This implies that the last letter of $\phi_{n}^{2}\left(\beta_{c+1}\right)$, which is $\phi_{n}^{2}\left(a_{r, n-1}\right)$, does not belong to $B_{n-1}^{+*}$. Then (1.7) implies $r=n-2$ and $\phi_{n}^{3}\left(a_{r, n-1}\right)=a_{1,2}$. As the normal form of $\beta_{c-1}$ is a word of $\underline{B}_{n-1}^{+*}$, the braid $\phi_{n}\left(\beta_{c-1}\right)$ can be represented by a word that contains no letter $a_{1, q}$. Now the relations

$$
a_{1,2} a_{p, q} \equiv \begin{cases}a_{p, q} a_{1,2} & \text { for } 2<p, \\ a_{1, q} a_{1,2} & \text { for } 2=p,\end{cases}
$$


imply that there exists a braid $\beta^{\prime}$ in $B_{n}^{+*}$ satisfying $a_{1,2} \phi_{n}\left(\beta_{c-1}\right) \equiv \beta^{\prime} a_{1,2}$. Therefore $a_{1,2}$ is a right-divisor of $\phi_{n}^{3}\left(\beta_{c+1}\right) \cdot \phi_{n}^{2}\left(\beta_{c}\right) \cdot \phi_{n}\left(\beta_{c-1}\right)$. As $c-1 \geq 2$ by hypothesis, this contradicts (2.9).

(iii) Assume that the normal form of $\beta_{k}$ is $w a_{n-2, n-1}$ with $w \neq \varepsilon$. Let $a_{p, q}$ be the last letter of $w$. As

$$
a_{p, q} a_{n-2, n-1} \equiv \begin{cases}a_{n-2, n-1} a_{p, q} & \text { for } q<n-2, \\ a_{p, n-1} a_{p, q} & \text { for } q=n-2,\end{cases}
$$

we must have $q=n-1$. Indeed, otherwise, $a_{p, q}$ would be a right-divisor of $\beta_{k}$, i.e., the $B_{n-1}^{+*}$-tail of $\phi_{n}\left(\beta_{k}\right)$ would be nontrivial, contradicting (2.9).

\subsection{Barriers}

If $\left(\beta_{b}, \ldots, \beta_{1}\right)$ is the $\phi_{n}$-splitting of a braid of $B_{n}^{+*}$, then Lemma 3.2 says that, for $k \geq 3$, the letter $\beta_{k}^{\#}$ must be some letter $a_{p-1, n-1}$. We shall see now that the braid $\beta_{k-1}$ cannot be an arbitrary braid of $B_{n-1}^{+*}$ : its normal form has to satisfy some constraints involving the integer $p$, namely containing a letter called an $a_{p, n}$-barrier-a key point in subsequent results.

Definition 3.3. The letter $a_{r, s}$ is called an $a_{p, n}$-barrier if

$$
1 \leq r<p<s \leq n-1 .
$$

There exists no $a_{p, n}$-barrier with $n \leq 3$; the only $a_{p, 4}$-barrier is $a_{1,3}$, which is an $a_{2,4^{-}}$ barrier.

By definition, if the letter $x$ is an $a_{p, n}$-barrier, then in the presentation of $B_{n}^{+*}$ there exists no relation of the form $a_{p, n} \cdot x=y \cdot a_{p, n}$ allowing one to push the letter $a_{p, n}$ to the right through the letter $x$ : so, in some sense, $x$ acts as a barrier. We shall prove now that (almost) every nonterminal entry $\beta_{k}$ of a splitting necessarily contains a barrier-a key point for what follows. The reason is simple: if there were no barrier in $\beta_{k}$, then the relations would enable one to push the last letter of $\phi_{n}^{2}\left(\beta_{k+1}\right)$ through $\phi_{n}\left(\beta_{k}\right)$ and incorporate it in $\beta_{k-1}$, contradicting the definition of a splitting.

Lemma 3.4. Assume that $n \geq 3, \beta$ is a braid of $B_{n-1}^{+*}$ and the $B_{n-1}^{+*}$-tail of $\phi_{n}\left(a_{p, n} \beta\right)$ is trivial for $p \leq n-2$. Then the normal form of $\beta$ is not the empty word and it contains an $a_{p, n}$-barrier.

Proof. We assume that the normal form $w$ of $\beta$ contains no $a_{p, n}$-barrier, and derive a contradiction. Let $w^{\prime}$ be the word $a_{p, n} w$ and let $X$ be the set of all letters $a_{q, r}$ with $p<r \leq n-1$. Write $w^{\prime}=u v$ where $v$ is the maximal suffix of $w$ containing letters from $X$ only. By hypothesis, the $B_{n-1}^{+*}$-tail of $\phi_{n}\left(\overline{w^{\prime}}\right)$ is trivial. Hence the word $w^{\prime}$ ends with $a_{q, n-1}$ for some $q$, i.e., $v$ is not empty. As the first letter of $w^{\prime}$ is $a_{p, n}$, which is not in $X$, the word $u$ is not empty. Let $a_{s, t}$ be the last letter of $u$. By the construction of $u$, the letter $a_{s, t}$ is either $a_{p, n}$ or satisfies $t \leq p$. In both cases, the braid $\phi_{n}\left(a_{s, t}\right)$ lies in $B_{n-1}^{+*}$. We shall now prove that $a_{s, t}$ quasi-commutes with $v$, i.e., there exists a word $v^{\prime}$ 
satisfying $a_{s, t} v \equiv v^{\prime} a_{s, t}$. Any letter $a_{q, r}$ occurring in $v$ is not an $a_{p, n}$-barrier, i.e., it satisfies $p \leq q<r \leq n-1$. Hence, by the relations

$$
a_{s, t} a_{q, r} \equiv\left\{\begin{array}{lll}
a_{q, r} a_{s, t} & \text { for } p<q \text { or } t<p & \text { by (1.3), } \\
a_{s, r} a_{s, t} & \text { for } q=t=p & \text { by (1.4), } \\
a_{r, t} a_{s, t} & \text { for } q=s=p & \text { by (1.4), }
\end{array}\right.
$$

the letter $a_{s, t}$ quasi-commutes with $v$. Then $\phi_{n}\left(a_{s, t}\right)$ is a right-divisor of $\phi_{n}\left(a_{p, n} \beta\right)$. This contradicts the hypothesis that the $B_{n-1}^{+*}$-tail of $\phi_{n}\left(a_{p, n} \beta\right)$ is trivial since the braid $\phi_{n}\left(a_{s, t}\right)$ belongs to $B_{n-1}^{+*}$.

We now show how Lemma 3.4 can be used in the context of a $\phi_{n}$-splitting.

Lemma 3.5. Let $\left(\beta_{b}, \ldots, \beta_{1}\right)$ be a $\phi_{n}$-splitting of some braid of $B_{n}^{+*}$ with $n \geq 3$. Then, for each $k$ in $\{b-1, \ldots, 2\}$ such that $\beta_{k+1}^{\#}$ is not $a_{n-2, n-1}$ (if any), the normal form of $\beta_{k}$ contains an $\phi_{n}\left(\beta_{k+1}^{\#}\right)$-barrier.

Proof. Condition (2.9) implies that the $B_{n-1}^{+*}$-tail of $\phi_{n}^{b-k+1}\left(\beta_{b}\right) \cdot \ldots \cdot \phi_{n}^{2}\left(\beta_{k+1}\right) \phi_{n}\left(\beta_{k}\right)$ is trivial. In particular the $B_{n-1}^{+*}$-tail of $\phi_{n}^{2}\left(\beta_{k+1}^{\#}\right) \phi_{n}\left(\beta_{k}\right)$ is trivial. Then Lemma 3.4 implies that the normal form of $\beta_{k}$ contains an $a_{p, n}$-barrier.

Example 3.6. Let us consider the braid $\beta$ whose normal form is

$$
a_{2,4} a_{1,3} a_{4,5} a_{2,4} a_{2,4} a_{3,5} a_{4,5}
$$

The $\phi_{5}$-splitting of $\beta$ is $\left(\beta_{4}, \beta_{3}, \beta_{2}, \beta_{1}\right)$ with

$$
\beta_{4}=a_{1,4}, \quad \beta_{3}=a_{1,4}, \quad \beta_{2}=a_{3,4} a_{1,3} a_{1,3} a_{2,4} a_{3,4} \quad \text { and } \quad \beta_{1}=1 .
$$

The letter $\beta_{4}^{\#}$ is $a_{1,4}$, hence by Lemma 3.5 the normal form of $\beta_{3}$ must contain an $a_{2,5^{-}}$ barrier; indeed, $a_{1,4}$ is an $a_{2,5}$-barrier. The letter $\beta_{3}^{\#}$ is $a_{1,4}$. Then, again by Lemma 3.5, the normal form of $\beta_{2}$ has to contain an $a_{2,5}$-barrier; indeed, the normal form of $\beta_{2}$ is $a_{3,4} a_{1,3} a_{1,3} a_{2,4} a_{3,4}$, which contains the $a_{2,5}$-barrier $a_{1,3}$.

\subsection{Ladders}

We have seen above in Lemma 3.4 that every normal word $w$ of $\underline{B}_{n-1}^{+*}$ such that the $B_{n-1}^{+*}$ tail of $\phi_{n}\left(a_{p, n} \bar{w}\right)$, with $p \leq n-2$, is trivial contains at least one $a_{p, n}$-barrier. We shall see now that, under the same hypotheses, $w$ contains not only one barrier, but even a sequence of overlapping barriers. Words containing such sequences are what we shall call ladders.

Definition 3.7. For $n \geq 3$, we say that a normal word $w$ is an $a_{p, n}$-ladder of height $h$ lent on $a_{q-1, n-1}$ if there exists a decomposition

$$
w=w_{0} x_{1} w_{1} \ldots w_{h-1} x_{h} w_{h}
$$

and a sequence $p=f(0)<f(1)<\cdots<f(h)=n-1$ such that 
(i) for each $k \leq h$, the letter $x_{k}$ is an $a_{f(k-1), n}$-barrier of the form $a_{\text {., } f(k) \text {, }}$

(ii) for each $k<h$, the word $w_{k}$ contains no $a_{f(k), n}$-barrier,

(iii) the last letter of $w$ is $a_{q-1, n-1}$.

By convention, any $a$-word whose last letter is $a_{q-1, n-1}$ is an $a_{n-1, n}$-ladder lent on $a_{q-1, n-1}$ and its height is 0 . There exist no $a_{p, n}$-barrier with $n \leq 3$, hence there exist only $a_{2,3}$-ladders in $\underline{B}_{3}^{+*}$.

The concept of a ladder is easily illustrated by representing the generators $a_{p, q}$ as a vertical line from the $p$ th line to the $q$ th line on an $n$-line stave. Then, for every $k \geq 0$, the letter $x_{k}$ looks like a bar of a ladder-see Figure 5 .

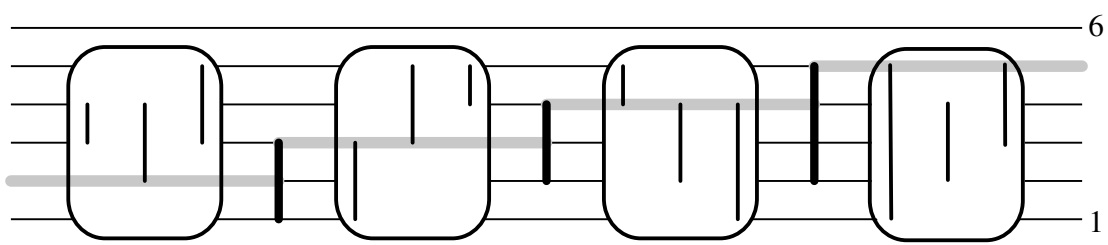

Fig. 5. An $a_{2,6}$-ladder lent on $a_{3,5}$ (the last letter). The gray line starts at position 2 and goes up to position 5 using the bars of the ladder. The empty spaces between bars in the ladder are represented by a framed box. In such boxes the vertical line representing the letter $a_{i, j}$ does not cross the gray line. The bars of the ladder are represented by black thick vertical lines.

Our aim is to prove that the normal form of each nonterminal entry in a $\phi_{n}$-splitting is a ladder. In order to do that, we begin with a preparatory lemma showing that barriers necessarily occur after certain letters of a normal form. Applying this result repeatedly will eventually provide us with a ladder.

Lemma 3.8. Assume that $n \geq 4, w$ is a suffix of a normal word of $\underline{B}_{n-1}^{+*}, a_{p, q}$ belongs to $B_{n-2}^{+*}$, and the $B_{n-1}^{+*}$-tail of $\phi_{n}\left(a_{p, q} \bar{w}\right)$ is trivial. Then $w$ contains an $a_{q, n}$-barrier.

Proof. (The method is formally similar to that for Lemma 3.4, but we repeat it because the technical details are actually different.) Let $X$ be the set of all letters $a_{r, s}$ with $s>q$. Write $a_{p, q} w=u v$ where $v$ is the maximal suffix containing letters of $X$ only. As, by hypothesis, the $B_{n-1}^{+*}$-tail of $\phi_{n}\left(a_{p, q} \bar{w}\right)$ is trivial, the last letter of $w$ exists and has the form $a_{., n-1}$, hence $v$ is nonempty.

As the letter $a_{p, q}$ does not lie in $X$, the word $u$ is not empty. Let $x=a_{t, t^{\prime}}$ be the last letter of $u$. By the definition of $u$, we have $t^{\prime} \leq q$. We suppose that $v$ contains no $a_{q, n^{-}}$ barrier, i.e., every letter $a_{r, s}$ of $v$ satisfies $r \geq q$, and eventually derive a contradiction. By (1.3) and (1.4), we have

$$
x a_{r, s} \equiv \begin{cases}a_{r, s} x & \text { for } r>q \text { or } t^{\prime}<q \\ a_{t, s} x & \text { for } q=r=t^{\prime}\end{cases}
$$

which implies that $x$ and $v$ quasi-commute, i.e., there exists an $a$-word $v^{\prime}$ satisfying $x v \equiv v^{\prime} x$. Then $\phi_{n}(x)$ is a right-divisor of the braid represented by $\phi_{n}\left(a_{p, q} w\right)$. The 
hypothesis about $a_{p, q}$ and the relation $t^{\prime} \leq q$ imply that $\phi_{n}(x)$ lies in $B_{n-1}^{+*}$, which contradicts the hypothesis that the $B_{n-1}^{+*}$-tail of $\phi_{n}\left(a_{p, q} \bar{w}\right)$ is trivial.

We can now show that every normal word satisfying some mild additional condition is a ladder.

Proposition 3.9. Assume that $n \geq 3, \beta$ belongs to $B_{n-1}^{+*}$ and the $B_{n-1}^{+*}$-tail of $\phi_{n}\left(a_{p, n} \beta\right)$ is trivial for some $p \leq n-2$. Then the normal form of $\beta$ is an $a_{p, n}$-ladder lent on $\beta^{\#}$.

Proof. We put $f(0)=p$ and let $w$ be the normal form of $\beta$. Lemma 3.5 implies that $w$ admits a decomposition $w_{0} x_{1} w^{(0)}$, where $w_{0}$ is the maximal prefix of $w$ that contains no $a_{p, n}$-barrier and $x_{1}=a_{., f(1)}$ is an $a_{p, n}$-barrier. By hypothesis, the $B_{n-1}^{+*}$-tail of the braid $\phi_{n}\left(a_{p, n} \bar{w}\right)$ is trivial, i.e., the $B_{n-1}^{+*}$-tail of $\phi_{n}\left(x_{1} \bar{w}^{(0)}\right)$ is trivial. Assume $f(1) \neq n-1$. Lemma 3.8 implies that the word $w^{(0)}$ admits a decomposition $w_{1} x_{2} w^{(1)}$, where $w_{1}$ is the maximal prefix of $w^{(0)}$ that contains no $a_{f(1), n}$-barrier and $x_{2}$ is an $a_{f(1), n}$-barrier. The same argument repeats until we find a decomposition $w_{0} x_{1} w_{1} \ldots x_{h} w^{(h-1)}$ with $f(h)=$ $n-1$. Then, putting $w_{h}=w^{(h-1)}$, we have obtained for $\beta$ an expression that satisfies all requirements of Definition 3.7.

Applying Proposition 3.9 to the successive entries of a $\phi_{n}$-splitting allows one to deduce that its entries contain ladders.

Corollary 3.10. Assume that $n \geq 3$ and $\left(\beta_{b}, \ldots, \beta_{1}\right)$ is a sequence in $B_{n-1}^{+*}$ that is the $\phi_{n}$-splitting of some braid of $B_{n}^{+*}$. Then, for each $k$ in $\{b-1, \ldots, 2\}$, the normal form of $\beta_{k}$ is a $\phi_{n}\left(\beta_{k+1}^{\#}\right)$-ladder lent on $\beta_{k}^{\#}$.

Proof. Condition (2.9) implies that the $B_{n-1}^{+*}$-tail of $\phi_{n}^{2}\left(\beta_{k+1}\right) \phi_{n}\left(\beta_{k}\right)$ is trivial. In particular, the $B_{n-1}^{+*}$-tail of $\phi_{n}^{2}\left(\beta_{k+1}^{\#}\right) \phi_{n}\left(\beta_{k}\right)$ is trivial. By Lemma 3.2, the letter $\beta_{k+1}^{\#}$ has the form $a_{., n-1}$. Then Proposition 3.9 implies that the normal form of $\beta_{k}$ is a $\phi_{n}\left(\beta_{k+1}^{\#}\right)$-ladder lent on $\beta_{k}^{\#}$.

For subsequent use it is worth noting that, by the definition of a ladder, as the letter $a_{n-2, n-1}$ is not a barrier, if a word $w a_{n-2, n-1}$ is an $a_{p, n}$-ladder and $w$ is nonempty, then $w$ is an $a_{p, n}$-ladder lent on $a_{r-1, n-1}$ for some $r$-see Lemma 3.2(iii).

Another consequence of Proposition 3.9 is:

Corollary 3.11. Assume that $n \geq 3$ and $\left(\beta_{b}, \ldots, \beta_{1}\right)$ is a sequence in $B_{n-1}^{+*}$ that is the $\phi_{n}$-splitting of some braid of $B_{n}^{+*}$. Then, for each $c$ in $\{b-1, \ldots, 2\}$ such that $\beta_{c}$ is either 1 or $a_{n-2, n-1}$, we have $\beta_{c+1}^{\#}=a_{n-2, n-1}$.

Proof. Assume $\beta_{c} \in\left\{1, a_{n-2, n-1}\right\}$. Let $a_{p-1, n-1}$ be the last letter of $\beta_{c+1}$. Condition (2.9) implies that the $B_{n-1}^{+*}$-tail of $\phi_{n}^{2}\left(\beta_{c+1}\right) \phi_{n}\left(\beta_{c}\right)$ is trivial. In particular the $B_{n-1}^{+*}$-tail of $\phi_{n}\left(a_{p, n} \beta_{c}\right)$ is trivial. Then, as the normal form of $\beta_{c}$ contains no barrier, Proposition 3.9 implies $p=n-1$. Therefore $\beta_{c+1}^{\#}=a_{n-2, n-1}$.

Example 3.12. Let us consider the braid of Example 3.6 again. Its $\phi_{4}$-splitting is is $\left(\beta_{4}, \ldots, \beta_{1}\right)$ with $\beta_{4}=a_{1,4}, \beta_{3}=a_{1,4}, \beta_{2}=a_{3,4} a_{1,3} a_{1,3} a_{2,4} a_{3,4}$ and $\beta_{1}=1$. The normal form of $\beta_{4}$ ends with $a_{1,4}$, hence the normal form of $\beta_{3}$ must be an $a_{2,5}$-ladder 
lent on $a_{1,4}$. Indeed, the ladder is $\varepsilon \cdot a_{1,4} \cdot \varepsilon$, and it has height 1 , corresponding, with the notation of Definition 3.7, to $w_{0}=\varepsilon, x_{1}=a_{1,4}$ and $w_{1}=\varepsilon$. Similarly, the normal form of $\beta_{3}$ ends with $a_{1,4}$, hence by Corollary 3.10, the normal form of $\beta_{2}$ must be an $a_{2,5}$-ladder lent on $a_{3,4}$. Indeed, here the ladder has height 2 , and its decomposition is $a_{3,4} \cdot a_{1,3} \cdot a_{1,3} \cdot a_{2,4} \cdot a_{3,4}$, corresponding, with the notation of Definition 3.7, to $w_{0}=a_{3,4}$, $x_{1}=a_{1,3}, w_{1}=a_{1,3}, x_{2}=a_{2,4}$ and $w_{2}=a_{3,4}$. We observe that $a_{1,3}$ is an $a_{2,5}$-barrier and $a_{2,4}$ is an $a_{3,5}$-barrier.

\section{Reversing}

In Section 3, we have established that almost every normal word is a ladder. We wish to use this result to establish Theorem 1, i.e., to obtain (short) $\sigma$-definite expressions. The basic question is as follows. Starting with a braid word that contains letters $\sigma_{i}$ with both positive and negative exponents, we shall try to obtain an equivalent word that is $\sigma$-positive-it is known that one cannot obtain both a $\sigma$-positive and a $\sigma$-negative expression, so our attempt must fail in some cases. The problem is to get rid of the letters $\sigma_{i}^{-1}$ with maximal index $i$. We shall see that, without loss of generality, we can assume that the initial word consists of an initial fragment - that will be called dangerous - containing negative letters (those with a negative exponent), followed by a normal word, hence by a ladder according to Proposition 3.9. Then the main technical step consists in proving that the product of a dangerous word with a ladder can be transformed using a simple algorithmic process called reversing into an equivalent $\sigma$-positive word: roughly speaking, ladders protect against dangerous elements.

\subsection{D-words}

Up to now, we have considered braid words involving letters of two different alphabets, namely the Artin generators $\sigma_{i}$ and the Birman-Ko-Lee generators $a_{p, q}$. From now on, we shall also use a third alphabet, corresponding to the following braids.

Definition 4.1. For $1 \leq p<q$, we put

$$
d_{p, q}=a_{p, p+1} a_{p+1, p+2} \ldots a_{q-1, q} \quad\left(=\sigma_{p} \sigma_{p+1} \ldots \sigma_{q-1}\right) .
$$

So, in particular, the equalities

$$
a_{p, q}=d_{p, q} d_{p, q-1}^{-1}=d_{p, q-1} \sigma_{q-1} d_{p, q-1}^{-1}
$$

hold for $1 \leq p<q$.

Hereafter it is convenient to use $d_{p, q}$ as a single letter. In this context, a word on the letters $d_{p, q}^{ \pm 1}$ (resp. $a_{p, q}^{ \pm 1}$ and $d_{p, q}^{ \pm 1}$, resp. $\sigma_{i}^{ \pm 1}$ ) will be called a $d$-word (resp. an $a d$-word, resp. a $\sigma$-word). We adopt the convention that the $d$-word $d_{p, p}$ is the empty word $\varepsilon$ for all $p$. 
All words over the above alphabets represent braids, and they can be translated into $\sigma$-words. It is coherent with the intended braid interpretations to define words $\underline{a}_{p, q}$ and $\underline{d}_{p, q}$ by

$$
\underline{a}_{p, q}=\sigma_{p} \ldots \sigma_{q-2} \sigma_{q-1} \sigma_{q-2}^{-1} \ldots \sigma_{p}^{-1}, \quad \underline{d}_{p, q}=\sigma_{p} \ldots \sigma_{q-1} .
$$

In this way, for each $a d$-word $w$, the braid represented by $w$ coincides with the braid represented by the $\sigma$-word $\underline{w}$ obtained from $w$ by replacing every letter $a_{p, q}$ by $\underline{a}_{p, q}$ and every letter $d_{p, q}$ by $\underline{d}_{p, q}$, and no ambiguity can result from using different alphabets. Of course, if $w$ and $w^{\prime}$ are two $a d$-words, we declare that $w \equiv w^{\prime}$ if the $\sigma$-words $\underline{w}$ and $\underline{w}^{\prime}$ are equivalent under the braid relations (1.1). Note in particular that the braid represented by the $d$-word $d_{1, n}$ is the Garside braid $\delta_{n}$.

The following equivalences of $a d$-words easily result from the definitions.

Lemma 4.2. The following relations are satisfied:

$$
\begin{aligned}
d_{p, r} & \equiv d_{p, q} d_{q, r} & & \text { for } p<q<r, \\
\phi_{n}\left(d_{p, q}\right) & \equiv d_{p+1, q+1} & & \text { for } p<q \leq n-1, \\
d_{p, q} d_{r, s} & \equiv d_{r, s} d_{p, q} & & \text { for } p<q<r<s, \\
d_{r, s}^{-1} a_{p, q} d_{r, s} & \equiv \phi_{s}^{-1}\left(\phi_{r}\left(a_{p, q}\right)\right) & & \text { for } p<q \leq r<s .
\end{aligned}
$$

Proof. Relation (4.3a) holds by the definition of $d_{p, q}$. Relation (4.3b) is an immediate consequence of (1.7). For (4.3c), we observe that the $\sigma_{i}$ of greatest index occurring in $d_{p, q}$ is $\sigma_{q-1}$, while the $\sigma_{i}$ of lower index occurring in $d_{r, s}$ is $\sigma_{r}$. As $q<r$ implies $q-1 \leq r-2$, we can apply Artin's commutativity relation (1.1) to obtain the expected result.

It remains to prove (4.3d). First, (4.3a) implies $d_{1, s} \equiv d_{1, r} d_{r, s}$, hence $d_{r, s} \equiv d_{1, r}^{-1} d_{1, s}$. We deduce $d_{r, s}^{-1} a_{p, q} d_{r, s} \equiv d_{1, s}^{-1} d_{1, r} a_{p, q} d_{1, r}^{-1} d_{1, s}$. As, by hypothesis, $a_{p, q}$ lies in $B_{r}^{+*}$, the subword $d_{1, r} a_{p, q} d_{1, r}^{-1}$ is equivalent to $\phi_{r}\left(a_{p, q}\right)$. Finally the conjunction of $B_{r}^{+*} \subseteq B_{s}^{+*}$ and $\phi_{r}\left(a_{p, q}\right) \in B_{r}^{+*}$ implies $d_{1, s}^{-1} \phi_{r}\left(a_{p, q}\right) d_{1, s} \equiv \phi_{s}^{-1}\left(\phi_{r}\left(a_{p, q}\right)\right)$.

\subsection{Sigma-positive words}

Our aim is to obtain $\sigma$-positive and $\sigma$-negative expression words. We shall need slightly more precise versions of these notions.

Definition 4.3. (i) $\mathrm{A} \sigma$-word $w$ is called $\sigma_{i}$-positive (resp. $\sigma_{i}$-negative) if $w$ contains at least one letter $\sigma_{i}$ and no letter $\sigma_{i}^{-1}$ (resp. at least one letter $\sigma_{i}^{-1}$ and no letter $\sigma_{i}$ ) and no letter $\sigma_{j}^{ \pm 1}$ for $j>i$.

(ii) A $\sigma$-word $w$ is said to be $\sigma_{i}$-nonnegative if it is either $\sigma_{i}$-positive, or contains no letter $\sigma_{j}^{ \pm 1}$ with $j \geq i$.

(iii) An $a d$-word $w$ is called $\sigma_{i}$-positive (resp. $\sigma_{i}$-negative, resp. $\sigma_{i}$-nonnegative) if the word $\underline{w}$ is $\sigma_{i}$-positive (resp. $\sigma_{i}$-negative, resp. $\sigma_{i}$-nonnegative).

Example 4.4. A $\sigma$-word cannot be simultaneously $\sigma_{i}$-positive and $\sigma_{i}$-negative, but, on the other hand, a $\sigma$-word can be neither $\sigma_{i}$-positive nor $\sigma_{i}$-negative for any $i$. For instance, 
$\sigma_{2} \sigma_{1} \sigma_{2}^{-1}$ is neither $\sigma_{2}$-positive (since it contains a letter $\sigma_{2}^{-1}$ ), nor $\sigma_{2}$-negative (since it contains a letter $\sigma_{2}$ ), nor $\sigma_{1}$-positive or $\sigma_{1}$-negative (since it contains a letter $\sigma_{2}$ ). By contrast, the equivalent word $\sigma_{1}^{-1} \sigma_{2} \sigma_{1}$ is $\sigma_{2}$-positive. On the other hand, the empty word, $\sigma_{1}^{-1}$, and $\sigma_{2} \sigma_{1}^{-1}$ are $\sigma_{2}$-nonnegative words, since the letter $\sigma_{2}^{-1}$ does not occur in them.

As for $a$-words, $a_{2,3}^{-1} a_{1,3}$ is not $\sigma_{2}$-positive, since its translation under (4.2) is the $\sigma$ word $\sigma_{2}^{-1} \sigma_{1} \sigma_{2} \sigma_{1}^{-1}$, which is not $\sigma_{2}$-positive as it contains the letter $\sigma_{2}^{-1}$. However, the previous $a$-word is equivalent to the $a$-word $a_{1,3} a_{1,2}^{-1}$, which translates into $\sigma_{1} \sigma_{2} \sigma_{1}^{-1} \sigma_{1}^{-1}$ and is therefore $\sigma_{2}$-positive, since $\sigma_{1} \sigma_{2} \sigma_{1}^{-1} \sigma_{1}^{-1}$ contains one letter $\sigma_{2}$ and no letter $\sigma_{2}^{-1}$.

An immediate consequence of Definition 4.3(iii) is

Lemma 4.5. An ad-word $w$ is $\sigma_{i}$-positive if $w$ contains at least one letter $a_{., i+1}$ or $d_{., i+1}$, and no letter $a_{., i+1}^{-1}, d_{., i+1}^{-1}, a_{., j}^{ \pm 1}$, or $d_{., j}^{ \pm 1}$ with $j>i+1$.

\subsection{Dangerous words}

We arrive at a key notion. The problem is to identify the generic form of the $\sigma$-negative fragments we wish to control and, possibly, get rid of. It turns out that the convenient notion is defined in terms of the letters $d_{p, q}^{-1}$, and it is what we call a dangerous word.

Definition 4.6. For $n \geq 3$, a $d$-word is called $a_{p, n}$-dangerous of type $q$ if it has the form

$$
d_{f(d), n-1}^{-1} d_{f(d-1), n-1}^{-1} \ldots d_{f(1), n-1}^{-1}
$$

with $q=f(d) \geq f(d-1) \geq \cdots \geq f(1)=p$.

By convention the unique $a_{n-1, n}$-dangerous word is the empty word.

Note that a dangerous $d$-word $w$ is completely determined by the $\sigma$-word $\underline{w}$. Indeed, we recover $w$ from $\underline{w}$ by gathering the $\sigma_{i}^{-1}$ 's and cutting before each letter $\sigma_{n-2}^{-1}$. For instance, $\sigma_{3}^{-1} \sigma_{2}^{-1} \sigma_{3}^{-1} \sigma_{2}^{-1} \sigma_{1}^{-1}$ can only be the translation of the $a_{1,5}$-dangerous word $d_{2,4}^{-1} d_{1,4}^{-1}$.

At this point, the definition of a dangerous word comes out of a hat. For the moment, let us observe that the letter $a_{p, n}$ is equivalent to $d_{p, n} d_{p, n-1}^{-1}$. In this expression, $d_{p, n-1}^{-1}$, which is $a_{p, n}$-dangerous, corresponds to the negative fragment of $a_{p, n}$. This reflects the intuition that dangerous words are associated with the negative parts of $a$-words-hence with their dangerous parts in view of our aim, which is to find $\sigma$-positive expressions.

\subsection{The reversing algorithm}

The aim of this section is to describe an algorithm that, starting with an $a_{p, n}$-dangerous word $u$ and an $a_{p, n}$-ladder $w$, returns a $\sigma_{n-2}$-positive word $w^{\prime}$ that is equivalent to $u w$ and that is close to being an $a_{p, n}$-ladder in a sense that will be defined below.

The basic ingredient is a process called reversing that transforms (certain) ad-words with letters $d_{., n-1}^{-1}$ on the left into equivalent words with letters $d_{., n-1}^{-1}$ on the right (or with no letter $d_{., n-1}^{-1}$ at all). Thus reversing is a process of pushing letters $d_{., n-1}^{-1}$ to the right. 
Definition 4.7. Let $w, w^{\prime}$ be $a d$-words. We write $w \curvearrowright^{(1)} w^{\prime}$ if $w^{\prime}$ is obtained from $w$ by replacing a subword $u$ of $w$ by a word $u^{\prime}$ such that $\left(u, u^{\prime}\right)$ is one of the pairs

$$
\begin{array}{ll}
\left(d_{p, n-1}^{-1} a_{r, s}, R_{p}\left(a_{r, s}\right) d_{p, n-1}^{-1}\right) & \text { with } s \leq p \leq n-2 \text { or } p \leq r \leq n-2, \\
\left(d_{p, n-1}^{-1} a_{r, s}, d_{r, n-1} R_{p}^{\prime}\left(a_{r, s}\right) d_{s, n-1}^{-1}\right) & \text { with } r<p<s \leq n-1, \\
\left(d_{p, n-1}^{-1} d_{r, n-1}, d_{r, n-1} R_{p}^{\prime \prime}\right) & \text { with } r<p \leq n-2,
\end{array}
$$

with

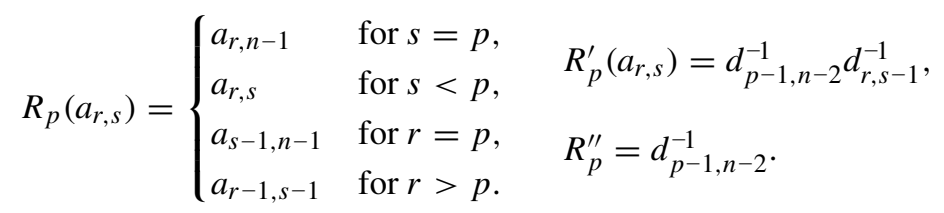

We say that $w$ reverses to $w^{\prime}$, denoted $w \curvearrowright w^{\prime}$, if there exists a sequence of words $w_{0}, w_{1}, \ldots, w_{\ell}$ satisfying $w_{0}=w, w_{\ell}=w^{\prime}$, and $w_{k} \curvearrowright^{(1)} w_{k+1}$ for every $k$.

Before giving an example, we introduce the notion of a reversing diagram, which enables one to conveniently illustrate the reversing process. Assume that $w_{0}, w_{1}, \ldots, w_{\ell}$ is a reversing sequence, i.e., is a sequence of $a d$-words such that $w_{k} \curvearrowright^{(1)} w_{k+1}$ for every $k$. First, we associate with $w_{0}$ a path labeled with the successive letters of $w_{0}$ : we associate to every letter $d_{p, n-1}^{-1}$ a vertical down-oriented edge labeled $d_{p, n-1}$, and to every other letter $x$ a horizontal right-oriented edge labeled $x$. Then we successively represent the words $w_{1}, \ldots, w_{\ell}$ as follows: if $w_{k+1}$ is obtained from $w_{k}$ by replacing $d_{p, n-1}^{-1} x$ by $u d_{q, n-1}^{-1}$ (where $d_{p, n-1}^{-1} x \curvearrowright^{(1)} u d_{q, n-1}^{-1}$ ), then we complete the pattern associated with the subword $d_{p, n-1}^{-1} x$ using right-oriented edges labeled $u$ and down-oriented edge labeled $d_{q, n-1}$; see Figure 6 .
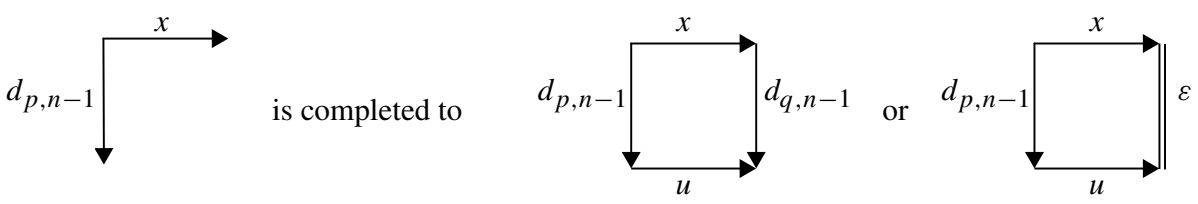

Fig. 6. Reversing of $d_{p, n-1}^{-1} x$ into $u d_{q, n-1}$. We replace the down-oriented edge labeled $d_{q, n-1}$ by a vertical double line labeled $\varepsilon$ whenever $q=n-1$, i.e., $d_{q, n-1} \equiv \varepsilon$.

Assume that $w$ and $w^{\prime}$ are $a d$-words and $w$ reverses to $w^{\prime}$. Then the reversing sequence going from $w$ to $w^{\prime}$ is not unique in general, but the resulting reversing diagram depends on $w$ and $w^{\prime}$ only. Reversing can easily be turned into a (deterministic) algorithm by choosing to always reverse the rightmost possible subword. The algorithm terminates when a word with no subword $d_{p, n-1}^{-1} x$ satisfying $d_{p, n-1}^{-1} x \curvearrowright u^{\prime}$ for some $u^{\prime}$ has been obtained. This algorithm is called reversing algorithm. See Figure 7 for an example. 


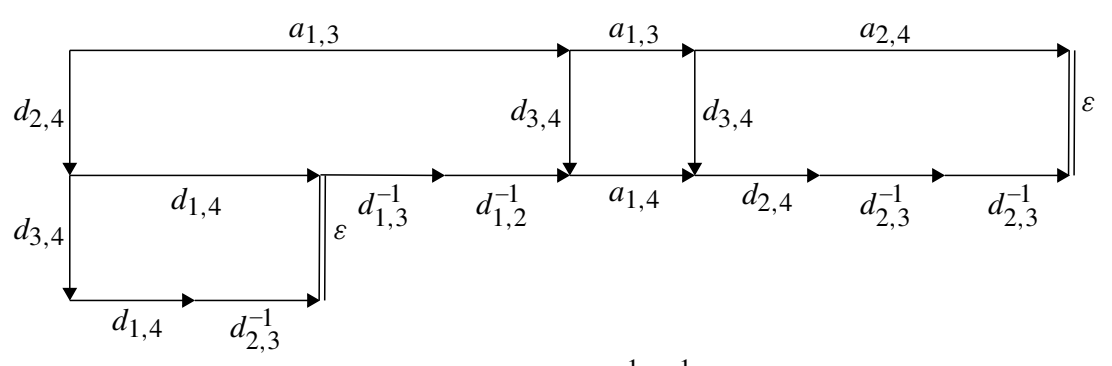

Fig. 7. Reversing diagram for the word $d_{3,4}^{-1} d_{2,4}^{-1} a_{1,3} a_{1,3} a_{2,4}$. We end up with $d_{1,4} d_{2,3}^{-1} d_{1,3}^{-1} d_{1,2}^{-1} a_{1,4} d_{2,4} d_{2,3}^{-1} d_{2,3}^{-1}$. Each rectangle in the diagram corresponds to one relation $u \curvearrowright^{(1)} u^{\prime}$, hence the number of rectangles is the length of every reversing sequence $\left(w_{0}, \ldots, w_{\ell}\right)$ from $w_{0}$ to $w_{\ell}$ : the sequence is not unique, but its length and the corresponding diagram are.

Remark 4.8. Formally, the above notion of reversing is similar to the transformation called "word reversing" in [9]. However, similarity is superficial only: what is common is the idea of iteratively pushing some specific factors to the right, but the factors considered and the basic switching rules are completely different.

The first, easy observation is that reversing transforms a braid word into an equivalent braid word.

Lemma 4.9. For $w, w^{\prime}$ ad-words, $w \curvearrowright w^{\prime}$ implies $w \equiv w^{\prime}$.

We skip the proof, which is a simple verification from the formulas of Lemma 4.2.

\section{Walls}

We shall now apply the reversing algorithm of Section 4.4 to those words that consist of an $a_{p, n}$-dangerous word followed by an $a_{p, n}$-ladder, with the aim of obtaining an equivalent $\sigma_{i}$-positive word whenever possible.

Once again, the problem is to identify the generic form of the final words we can obtain. A new type of braid word called wall occurs here, and the main result is that reversing a word consisting of a dangerous word followed by a ladder always results in a $\sigma$-nonnegative word that is a wall.

\subsection{Dangerous against ladders: case of length 1}

We first concentrate on the case when the dangerous word has length 1, i.e., it consists of a single negative $d$-letter-the general case will be handled in Section 5.3. In view of Theorems 1 and 2, we shall not only describe the resulting $a d$-word, but also compute both the time and space complexity of the algorithm involved in the transformation.

First we introduce the notion of a wall, a weak variant of a ladder. It comes in two versions called high and low. 
Definition 5.1. For $n \geq 3$ and $p \leq n-2$, we say that an $a d$-word $w$ is a high $a_{p, n}$-wall lent on $a_{q-1, n-1}$ if there exists a decomposition

$$
w=u \cdot d_{r, n-1} \cdot w^{\prime} \cdot d_{q-1, n-1} \cdot v
$$

such that

- $u$ is a positive $a$-word,

- $r<p$,

- $w^{\prime}$ is a $\sigma_{n-2}$-nonnegative $a d$-word,

- $v$ is $a_{q-1, n-1}$-dangerous.

We say that an $a d$-word $w$ is a low $a_{p, n}$-wall lent on $a_{q-1, n-1}$ if there exists a decomposition

$$
w=u \cdot d_{q-1, n-1} \cdot v
$$

such that

- $u$ is a positive $a$-word,

- $q-1<p$,

- $v$ is $a_{q-1, n-1}$-dangerous of type $p^{\prime}<p$.

In both cases, we write $F(w)$ for the word denoted $u$ above, and $D(w)$ for the word denoted $v$ above.

We say that an $a$-word $w$ is an $a_{p, n}$-wall if it is either a high or a low $a_{p, n}$-wall.

Note that the condition satisfied by the letter $d_{r, n-1}$ occurring in the decomposition of a high wall is the condition satisfied by the $a_{p, n}$-barrier $a_{r, n-1}$. The same property holds for the letter $d_{q-1, n-1}$ occurring in the decomposition of a low wall.

So far we have defined $a_{p, n}$-walls for $p \leq n-2$ only. We now consider $a_{n-1, n}$-walls, which are special as are $a_{n-1, n}$-ladders.

Definition 5.2. For $n \geq 3$, we say that an $a d$-word $w$ is an $a_{n-1, n}$-wall lent on $a_{q-1, n-1}$ if $w$ can be decomposed as $u \cdot d_{q-1, n-1} \cdot v$ with $u$ a positive $a$-word and $v$ an $a_{q-1, n-1^{-}}$ dangerous word. Then we define $F(w)=u$ and $D(w)=v$.

By definition, every $a_{p, n}$-wall lent on $a_{q-1, n-1}$ is also an $a_{r, n}$-wall lent on $a_{q-1, n-1}$ for $r \geq p$.

Walls are introduced in order to describe the output of the reversing algorithm running on those words that consist of an $a_{p, n}$-dangerous word followed by an $a_{p, n}$-ladder.

Lemma 5.3. Let $w$ be an $a_{p, n}$-ladder lent on $a_{q-1, n-1}$ with $p \leq n-2$ and $n \geq 3$. Let $w_{0} x_{1} \ldots x_{h} w_{h}$ be the decomposition of $w$ as a ladder. Then $d_{p, n-1}^{-1} w$ is equivalent to an $a_{p, n}$-wall $w^{\prime}$ lent on $a_{q-1, n-1}$. The latter can be computed using at most $|w|$ reversing steps plus one basic operation, and it satisfies

- $\left|F\left(w^{\prime}\right)\right|=\left|w_{0}\right|$,

- $\left|D\left(w^{\prime}\right)\right| \leq 2$,

- $\left|w^{\prime}\right| \leq|w|+2(h-1)+2\left|w_{h}\right|+\left|D\left(w^{\prime}\right)\right|$

- $w^{\prime}$ is a high wall for $w_{h} \neq \varepsilon$. 


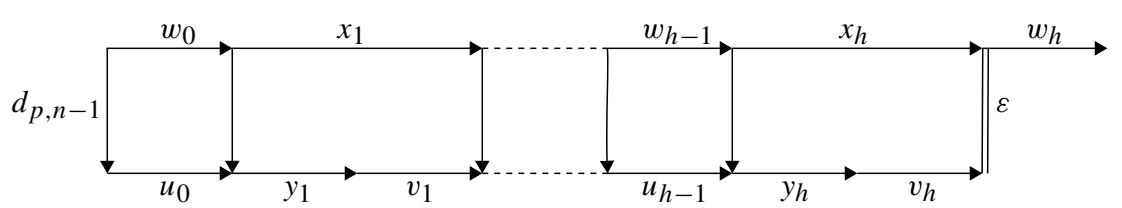

Fig. 8. Reversing $d_{p, n-1}^{-1} w$ into a wall when $w$ is a ladder (proof of Lemma 5.3).

Proof. The main idea is illustrated in Figure 8: starting with $d_{p, n-1}^{-1} w$, i.e., with $d_{p, n-1}^{-1} w_{0} x_{1} \ldots x_{h} w_{h}$, we reverse the diagram by pushing the vertical (negative) $d$-arrows to the right until a wall is obtained. The success at each elementary step is guaranteed by Lemma 4.9. In general we obtain a high wall. A few particular cases have to be considered separately, namely when $w_{h}$ is empty, in which case we obtain a low wall if the height $h$ is 1 .

We start with a description of elementary blocks of the diagram of Figure 8. Write $x_{k}=a_{e(k), f(k)}$ for $k=1, \ldots, h$, and put $f(0)=p$. Fix $k$ with $0 \leq k \leq h-1$. Put $u_{k}=$ $R_{f(k)}\left(w_{k}\right), y_{k+1}=d_{e(k+1), n-1}$ and $v_{k+1}=R_{f(k)}^{\prime}\left(x_{k+1}\right)$. Then we have $d_{f(k), n-1}^{-1} w_{k} x_{k+1} \curvearrowright$ $u_{k} y_{k+1} v_{k+1} d_{f(k+1), n-1}^{-1}$, corresponding to the diagram

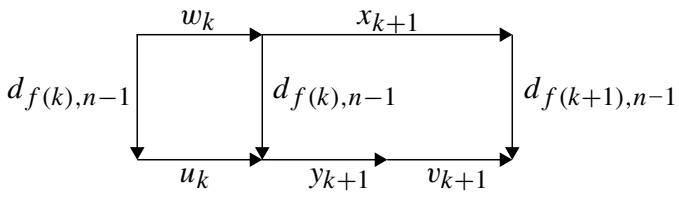

Gathering the reversing diagrams corresponding to the successive values of the parameter $k$, we precisely obtain the diagram of Figure 8. Put $w_{k}^{\prime}=u_{k} y_{k+1} v_{k+1}$ for $0 \leq k \leq h-1$.

At this point, we have to consider three slightly different cases. Assume first $w_{h}=\varepsilon$ and $h \geq 2$, the easiest case, from which the other two cases will be derived.

Put $w^{\prime}=w_{0}^{\prime} \ldots w_{h-1}^{\prime}$. By construction, we have $d_{p, n-1}^{-1} w \curvearrowright w^{\prime}$. Hence, by Lemma $4.9, d_{p, n-1}^{-1} w$ is equivalent to $w^{\prime}$. We shall now prove that $w^{\prime}$ is a wall of the expected type, and that the complexity statements are satisfied.

As $w_{h}$ is empty, the last letter of $w$ is $x_{h}$. This implies $x_{h}=a_{q-1, n-1}$, hence $y_{h}=$ $d_{q-1, n-1}$. Put $w^{\prime \prime}=v_{1} w_{1}^{\prime} \ldots w_{h-2}^{\prime} u_{h-1}$. We recall that $w_{k}^{\prime}$ is equal to $u_{k} y_{k+1} v_{k+1}$. By construction, we have

$$
w^{\prime}=u_{0} \cdot d_{e(1), n-1} \cdot w^{\prime \prime} \cdot d_{q-1, n-1} \cdot v_{h} .
$$

We shall now check that $w^{\prime}$ is a high $a_{p, n}$-wall lent on $a_{q-1, n-1}$. As the image of an $a$ letter under $R_{p}$ is an $a$-letter, the word $u_{0}$ is a positive $a$-word whose length is $\left|w_{0}\right|$. Hence (5.1a) and (5.3a) are satisfied.

Next, by the definition of a ladder, the letter $x_{1}$ is an $a_{p, n}$-barrier, hence $e(1)<p$ holds, i.e., (5.1b) is satisfied. 
As the words $u_{k}, y_{k+1}, v_{k+1}$ are $\sigma_{n-2}$-nonnegative, the word $w^{\prime \prime}$ is also $\sigma_{n-2}$ nonnegative. So (5.1c) holds.

Now, we recall that $v_{h}$ is equal to $d_{f(h-1)-1, n-2}^{-1} d_{e(h), n-2}^{-1}$ with $e(h)=q-1$. By the definition of a ladder, the letter $x_{h}$ is an $a_{f(h-1), n}$-barrier. Therefore, we have $q-1<$ $f(h-1)$, which implies $f(h-1)-1 \geq q-1$. Hence $v_{h}$ is $a_{q-1, n-1}$-dangerous of length 2. So (5.1d) and (5.3b) are satisfied.

Finally, for $(5.3 \mathrm{c})$, we compute

$$
\left|w_{k}^{\prime}\right|=\left|u_{k}\right|+\left|y_{k+1}\right|+\left|v_{k+1}\right|=\left|w_{k}\right|+1+2=\left|w_{k} x_{k+1}\right|+2 .
$$

Then, as $w_{h}$ is empty, we obtain

$$
\left|w^{\prime}\right|=\sum_{k=0}^{h-1}\left|w_{k}^{\prime}\right|=\sum_{k=0}^{h-1}\left|w_{k} x_{k+1}\right|+2 h=|w|+2 h .
$$

As in this case $w_{h}$ is empty and the length of $D\left(w^{\prime}\right)$ is 2, i.e., the length of $v_{h}$ is 2 , condition (5.3c) holds. So the case of $w_{h}$ empty with $h \geq 2$ is completed, except for the time complexity analysis.

Assume now $w_{h}=\varepsilon$ and $h=1$. Then $w^{\prime}$ is equal to $u_{0} \cdot d_{q-1, n-1} \cdot v_{1}$. As in the previous case, the word $u_{0}$ is a positive $a$-word of length $w_{0}$ and we have $\left|w^{\prime}\right|=|w|+2$. The word $v_{1}$ is equal to $d_{p-1, n-2}^{-1} d_{q-1, n-2}^{-1}$, which is $a_{q-1, n-1}$-dangerous of type $p-1$ and has length 2 . Therefore, $w^{\prime}$ is a low $a_{p, n}$-wall lent on $a_{q-1, n-1}$ satisfying (5.3a), (5.3b) and $(5.3 \mathrm{c})$.

Assume finally $w_{h} \neq \varepsilon$. Then we decompose $w_{h}$ as $w_{h}^{\prime \prime} a_{q-1, n-1}$. Put

$$
w^{\prime}=w_{0}^{\prime} \ldots w_{h-1}^{\prime} w_{h}^{\prime \prime} d_{q-1, n-1} d_{q-1, n-2}^{-1}
$$

and $w^{\prime \prime}=v_{1} w_{1}^{\prime} \ldots w_{h-1}^{\prime} w_{h}^{\prime \prime}$. We have $w^{\prime}=u_{0} \cdot d_{e(1), n-1} \cdot w^{\prime \prime} \cdot d_{q-1, n-1} \cdot d_{q-1, n-2}^{-1}$. Then (5.1a), (5.1b), (5.1c) are checked as in the case $w_{h}=\varepsilon$. By construction, the word $d_{q-1, n-2}^{-1}$ is $a_{q-1, n-1}$-dangerous of length at most 1 . We recall that we use the convention that the $d$-word $d_{n-2, n-2}$ is the empty word $\varepsilon$. So (5.1d) and (5.3b) are satisfied. Then, by the definition of $w^{\prime},(5.3 \mathrm{~d})$ holds. We check now (5.3c). Starting from $\left|w_{k}^{\prime}\right|=\left|w_{k} x_{k+1}\right|+2$, we obtain

$$
\left|w^{\prime}\right| \leq \sum_{k=0}^{h-1}\left|w_{k}^{\prime}\right|+\left|w_{h}^{\prime \prime}\right|+2=\sum_{k=0}^{h-1}\left|w_{k} x_{k+1}\right|+\left|w_{h}\right|+2 h+1=|w|+2 h+1 .
$$

As $w_{h}$ is not empty, we have $\left|w_{h}\right| \geq 1$, hence $2\left|w_{h}\right| \geq 2$. Moreover, in this case, the length of $D\left(w^{\prime}\right)$ is at most 1 . Therefore, we get $3 \leq 2\left|w_{h}\right|+\left|D\left(w^{\prime}\right)\right|$, and eventually

$$
\left|w^{\prime}\right| \leq|w|+2(h-1)+2\left|w_{h}\right|+\left|D\left(w^{\prime}\right)\right| \text {. }
$$

So, all cases have been considered. It only remains to consider the time complexity. In the first and second cases, at most $|w|$ reversing operations are needed. In the last case$w_{h} \neq \varepsilon$-at most $|w|$ reversing operations are needed, plus the decomposition of $w_{h}^{\#}$ into at most two $d$-letters. 
Example 5.4. We saw in Example 3.12 that the word $w=a_{3,4} a_{1,3} a_{1,3} a_{2,4} a_{3,4}$ is an $a_{2,5^{-}}$ ladder lent on $a_{3,4}$. Let us compute the $a_{2,5}$-wall lent on $a_{3,4}$ that is equivalent to $d_{2,5}^{-1} w$. Applying the reversing algorithm to $d_{2,4}^{-1} w$ gives

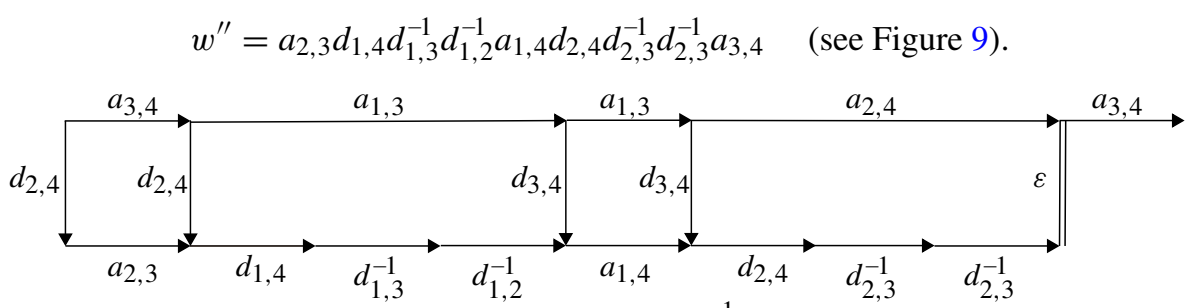

Fig. 9. Reversing diagram for the word $d_{2,4}^{-1} a_{3,4} a_{1,3} a_{1,3} a_{2,4} a_{3,4}$.

The word $w^{\prime \prime}$ is not a wall because its last letter does not have the correct form. However, if we replace the last letter $a_{3,4}$ of $w^{\prime \prime}$ by $d_{3,4}$ we obtain the high wall

$$
w^{\prime}=a_{2,3} \cdot d_{1,4} \cdot d_{1,3}^{-1} d_{1,2}^{-1} a_{1,4} d_{2,4} d_{2,3}^{-1} d_{2,3}^{-1} \cdot d_{3,4} \cdot \varepsilon .
$$

The word $F\left(w^{\prime}\right)$ of $w^{\prime}$ is $a_{2,3}$, whereas $D\left(w^{\prime}\right)$ is empty.

\subsection{Dangerous against wall}

In the previous section, we studied the action of the reversing algorithm running on a word $u w$ in the special case when $u$ is an $a_{p, n}$-dangerous word of length 1 and $w$ is an $a_{p, n}$-ladder. We proved that the output word is an $a_{p, n}$-wall. Before turning to the general case of an initial dangerous word with an arbitrary length - that will be done in the next section-we consider here the case of an $a_{p, n}$-dangerous word of length 1 followed by an $a_{p, n}$-wall. The result is that the output word is again an $a_{p, n}$-wall. This shows that, in contrast to the family of ladders, the family of walls enjoys good closure properties that will make inductive arguments possible.

We start with a technical result that will be used twice in the proof of Lemma 5.6.

Lemma 5.5. Assume that $n \geq 3, w$ is a positive $a$-word containing an $a_{p, n}$-barrier and $r<p$. Then the word $d_{p, n-1}^{-1} w d_{r, n-1}$ reverses to the ad-word

$$
u d_{t, n-1} u^{\prime} d_{r, n-1} d_{s-1, n-2}^{-1},
$$

which is obtained in at most $|w|+1$ steps and satisfies

- $t<p$ and $r<s$,

- $u$ is a positive a-word with $|u|<|w|$,

- $u^{\prime}$ is a $\sigma_{n-2}$-nonnegative ad-word,

- $\left|u d_{t, n-1} u^{\prime} d_{r, n-1} d_{s-1, n-2}^{-1}\right| \leq\left|w d_{r, n-1}\right|+2|w|-2|u|+1$.

Proof. Write $w$ as $w^{\prime} a_{t, t^{\prime}} v$ where $w^{\prime}$ is the maximal prefix of $w$ that contains no $a_{p, n^{-}}$ barrier, and with $a_{t, t^{\prime}}$ an $a_{p, n}$-barrier. The argument is illustrated in Figure 10: starting with $d_{p, n-1}^{-1} w d_{r, n-1}$, we reverse the diagram by pushing the vertical (negative) $d$-arrows to the right until a wall is obtained. The success at each elementary step is guaranteed by Lemma 4.9. 


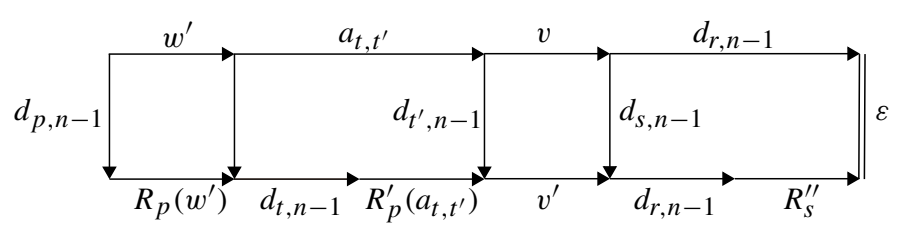

Fig. 10. Reversing diagram for the word $d_{p, n-1}^{-1} w d_{r, n-1}$ (proof of Lemma 5.5).

As $w^{\prime}$ does not contain any $a_{p, n}$-barrier, we have $d_{p, n-1}^{-1} w^{\prime} \curvearrowright R_{p}\left(w^{\prime}\right) d_{p, n-1}^{-1}$. By construction $a_{t, t^{\prime}}$ is an $a_{p, n}$-barrier, i.e., $t<p<t^{\prime}$. We deduce

$$
d_{p, n-1}^{-1} a_{t, t^{\prime}} \curvearrowright d_{t, n-1} R_{p}^{\prime}\left(a_{t, t^{\prime}}\right) d_{t^{\prime}, n-1}^{-1} .
$$

By the definition of elementary reversing steps, we obtain $d_{t^{\prime}, n-1}^{-1} v \curvearrowright v^{\prime} d_{s, n-1}^{-1}$ for some $a d$-word $v^{\prime}$ with $\left|v^{\prime}\right| \leq 3|v|$ and some $s \geq t^{\prime}$. The hypothesis $r<p$ together with $p<t^{\prime}$ and $t^{\prime} \leq s$ implies $r<s$. Hence $d_{s, n-1}^{-1} d_{r, n-1} \curvearrowright d_{r, n-1} R_{s}^{\prime \prime}$.

Write $u=R_{p}\left(w^{\prime}\right)$ and $u^{\prime}=R_{p}^{\prime}\left(a_{t, t^{\prime}}\right) v^{\prime}$. By construction, we have

$$
d_{p, n-1}^{-1} w d_{r, n} \curvearrowright u d_{t, n-1} u^{\prime} d_{r, n-1} d_{s-1, n-2}^{-1},
$$

and we claim that the latter word has the expected properties.

Condition (5.4a) is an immediate consequence of the above results.

As the image of an $a$-letter under $R_{p}$ is an $a$-letter, the word $u$ is a positive $a$-word of length $\left|w^{\prime}\right|$. By definition, $w^{\prime}$ is a proper prefix of $w$. Then $\left|w^{\prime}\right|<|w|$, i.e., (5.4b) is satisfied.

By the definition of elementary reversing steps, the image of a positive $a$-word under $R$ and $R^{\prime}$ is $\sigma_{n-2}$-nonnegative, hence $v^{\prime}$ is $\sigma_{n-2}$-nonnegative. As $R_{p}^{\prime}\left(a_{t, t^{\prime}}\right)$ is $\sigma_{n-2}-$ nonnegative, $u^{\prime}$ is $\sigma_{n-2}$-nonnegative, i.e., (5.4c) holds.

For $(5.4 \mathrm{~d})$, we compute

$$
\left|u d_{t, n-1} u^{\prime} d_{r, n-1} d_{s-1, n-2}^{-1}\right|=\left|w^{\prime}\right|+\left|u^{\prime}\right|+3=\left|w^{\prime}\right|+\left|v^{\prime}\right|+5 .
$$

By the construction of $v^{\prime}$, we have $\left|v^{\prime}\right| \leq 3|v|$. Using $|w|=\left|w^{\prime}\right|+1+|v|$, we deduce

$$
\left|u d_{t, n-1} u^{\prime} d_{r, n-1} d_{s-1, n-2}^{-1}\right| \leq 3|w|-2\left|w^{\prime}\right|+2=\left|w d_{r, n-1}\right|+2\left(|w|-\left|w^{\prime}\right|\right)+1,
$$

which is the expected inequality since $\left|w^{\prime}\right|=|u|$ by (5.4b).

An easy bookkeeping argument gives the bound on the number of steps in the reversing process.

We are now able to establish the main result of this section.

Lemma 5.6. Assume that $w$ is an $a_{p, n}$-wall lent on $a_{q-1, n-1}$. Then $d_{p, n-1}^{-1} w$ reverses in at most $|F(w)|+1$ steps to an $a_{p, n}$-wall $w^{\prime}$ satisfying

- $\left|F\left(w^{\prime}\right)\right| \leq|F(w)|$,

- $\left|D\left(w^{\prime}\right)\right| \leq|D(w)|+1$,

- $\left|w^{\prime}\right| \leq|w|+2|F(w)|-2\left|F\left(w^{\prime}\right)\right|+1$,

- $w^{\prime}$ is a high wall whenever $w$ is a high wall. 
Proof. Assume that $w$ is a low wall. Then $w$ admits the decomposition $w=$ $F(w) d_{q-1, n-1} D(w)$. By the definition of a wall, we have $q-1<p$. First, assume in addition that $F(w)$ contains no $a_{p, n}$-barrier. Then the reversing process gives

$$
d_{p, n-1}^{-1} w \curvearrowright R_{p}(F(w)) d_{p, n-1}^{-1} d_{q-1, n-1} D(w) \curvearrowright R_{p}(F(w)) d_{q-1, n-1} d_{p-1, n-2}^{-1} D(w) .
$$

Write $w^{\prime}=u \cdot d_{q-1, n-1} \cdot v$ with $u=R_{p}(F(w))$ and $v=d_{p-1, n-2}^{-1} D(w)$. As the image of a positive $a$-letter under $R_{p}$ is a positive $a$-letter, the word $u$ is a positive $a$-word of length $|F(w)|$. Then $q-1<p$ implies that $w^{\prime}$ is a low $a_{p, n}$-wall lent on $a_{q-1, n-1}$ satisfying (5.5a) and (5.5b) because $D\left(w^{\prime}\right)=v$. Condition (5.5c) is a direct consequence of the construction of $w^{\prime}$ together with $|v|=|D(w)|+1$.

Next, assume in addition that $F(w)$ contains an $a_{p, n}$-barrier. By Lemma 5.5 applied to $F(w) d_{q-1, n-1}$, there exist two words $u$ and $u^{\prime}$ and two integers $s$ and $t$ satisfying

$$
d_{p, n-1}^{-1} w \curvearrowright u d_{t, n-1} u^{\prime} d_{q-1, n-1} d_{s-1, n-2}^{-1} D(w) .
$$

Write $w^{\prime}=u \cdot d_{t, n-1} \cdot u^{\prime} \cdot d_{q-1, n-1} \cdot v$, with $v=d_{s^{-1, n-2}}^{-1} D(w)$. Condition (5.4a) implies that $v$ is an $a_{q-1, n-1}$-dangerous word of length at most $|D(w)|+1$, and that $t<p$ holds. Then, (5.4b) and (5.4c) imply that $w$ is a high $a_{p, n}$-wall lent on $a_{q-1, n-1}$ and it satisfies (5.5a) and (5.5b). Using (5.4d), we compute

$$
\left|w^{\prime}\right|=\left|F(w) d_{q-1, n-1}\right|+2|F(w)|-2|u|+|v|,
$$

which implies (5.5c) since we have $F\left(w^{\prime}\right)=u$ and $D\left(w^{\prime}\right)=d_{s-1, n-2}^{-1} D(w)=v$.

Assume now that $w$ is a high wall. Then $w$ admits the decomposition

$$
w=F(w) d_{r, n-1} w^{\prime \prime} d_{q-1, n-1} D(w),
$$

with $r<p$. First, assume that $F(w)$ contain no $a_{p, n}$-barrier. Then the reversing process gives

$$
d_{p, n-1}^{-1} w \curvearrowright R_{p}(F(w)) d_{r, n-1} d_{p-1, n-2}^{-1} w^{\prime \prime} d_{q-1, n-1} D(w) .
$$

Write $w^{\prime}=R_{p}(F(w)) \cdot d_{r, n-1} \cdot d_{p-1, n-2}^{-1} w^{\prime \prime} \cdot d_{q-1, n-1} \cdot D(w)$. A direct verification, based on the fact that $w$ is a high $a_{p, n}$-wall lent on $a_{q-1, n-1}$, gives that $w^{\prime}$ is a high $a_{p, n}$-wall lent on $a_{q-1, n-1}$ satisfying (5.5a), (5.5b) and (5.5d). For (5.5c), we compute $\left|w^{\prime}\right|=|w|+1$.

Assume now that $F(w)$ contains an $a_{p, n}$-barrier. Then, by Lemma 5.5 applied to $F(w) d_{r, n-1}$, there exist two words $u, u^{\prime}$ and two integers $s, t$ satisfying

$$
d_{p, n-1}^{-1} w \curvearrowright u d_{t, n-1} u^{\prime} d_{r-1, n-1} d_{s-1, n-2}^{-1} w^{\prime \prime} d_{q-1, n-1} D(w) .
$$

Write $w^{\prime}=u \cdot d_{t, n-1} \cdot u^{\prime} d_{r-1, n-1} d_{s-1, n-2}^{-1} w^{\prime \prime} \cdot d_{q-1, n-1} \cdot D(w)$. Condition (5.4c) implies that the word $u^{\prime} d_{r-1, n-1} d_{s-1, n-2}^{-1} w^{\prime \prime}$ is $\sigma_{n-2}$-nonnegative, and even $\sigma_{n-2}$-positive. Then, a direct verification, based on the fact that $w$ is a high $a_{p, n}$-wall lent on $a_{q-1, n-1}$, shows that $w^{\prime}$ is a high $a_{p, n}$-wall lent on $a_{q-1, n-1}$ and it satisfies (5.5a), (5.5b) and (5.5d). Using (5.4d), we compute

$$
\left|w^{\prime}\right| \leq\left|F(w) d_{r, n-1}\right|+2|F(w)|-2|u|+1+\left|w^{\prime \prime}\right|+1+|D(w)|,
$$

which implies $(5.5 \mathrm{c})$ since $F\left(w^{\prime}\right)=u$.

As for the number of reversing steps, it follows from an easy bookkeeping argument using Lemma 5.5. 


\subsection{Dangerous against ladders: the general case}

In the previous section, we studied the action of the reversing algorithm running on a word $u w$ in the special case when $u$ is $a_{p, n}$-dangerous of length 1 and $w$ is an $a_{p, n}$-ladder. We proved that the output word is an $a_{p, n}$-wall. The aim of this section is to describe the reversing algorithm in the general case, i.e., for a dangerous word of arbitrary length.

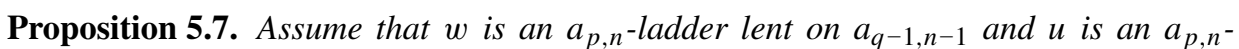
dangerous word of type $t$ with $n \geq 3$. Then $u w$ is equivalent to an $a_{t, n}$-wall $w^{\prime}$ lent on $a_{q-1, n-1}$ which can be computed using at most $|u||w|$ reversing steps, plus one basic operation, hence in time $O(|u||w|+1)$, and it satisfies

- $\left|D\left(w^{\prime}\right)\right| \leq|u|+1$,

- $\left|w^{\prime}\right| \leq 3|w|+|u|-1$.

Moreover, if $w$ is an $a_{p, n}$-ladder lent on $a_{n-2, n-1}$ but different from $a_{n-2, n-1}$, then $w^{\prime}$ admits the decomposition $w^{\prime}=w^{\prime \prime} d_{n-2, n-1}$, where $w^{\prime \prime}$ is a $\sigma_{n-2}$-positive word.

Proof. All ladders and walls in this proof are supposed to be lent on $a_{q-1, n-1}$. We shall construct an $a_{p, n}$-wall $w^{\prime}$ that is equivalent to $u w$ by induction on the length of $u$.

Assume first $p \leq n-2$. Then $u$ is not empty. Write $u$ as $d_{f(d), n-1}^{-1} \cdot \ldots \cdot d_{f(1), n-1}^{-1}$. By the definition of a dangerous word, we have $f(1)=p$ and $f(d)=t$. Define $w_{(1)}$ to be the $a_{f(1), n}$-wall provided by Lemma 5.3 that is equivalent to $d_{f(1), n-1}^{-1} w$. Starting from $w_{(1)}$, we inductively define $w_{(k+1)}$ to be the $a d$-word obtained by reversing $d_{f(k+1), n-1}^{-1} w_{(k)}$.

We claim that $w_{(k)}$ is an $a_{f(k), n}$-wall. Indeed, by the definition of a wall, the relation

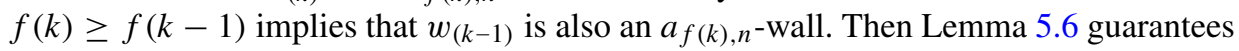
that $w_{(k)}$ is an $a_{f(k), n}$-wall.

By construction, we have $u w \equiv w_{(d)}$. In particular, $u w$ is an $a_{t, n}$-wall. We shall now prove that $w_{(d)}$ satisfies the complexity statements.

Lemma 5.3 gives $\left|D\left(w_{(1)}\right)\right| \leq 2$. For $1 \leq k \leq d-1$, (5.5b) implies $\left|D\left(w_{(k+1)}\right)\right| \leq$ $\left|D\left(w_{(k)}\right)\right|+1$. Hence, $\left|D\left(w_{(d)}\right)\right| \leq|u|+1$, i.e., (5.6a) is satisfied.

Let $w_{0} x_{1} \ldots x_{h} w_{h}$ be the decomposition of the $a_{p, n}$-ladder $w$. Then, by (5.5c), for each $k \geq 1$ we have

$$
\left|w_{(k+1)}\right| \leq\left|w_{(k)}\right|+2\left|F\left(w_{(k)}\right)\right|-2\left|F\left(w_{(k+1)}\right)\right|+1 .
$$

Gathering the various relations $(5.7)$ for $k=1, \ldots, d-1$, we obtain

$$
\left|w_{(d)}\right| \leq\left|w_{(1)}\right|+2\left|F\left(w_{(1)}\right)\right|-2\left|F\left(w_{(d)}\right)\right|+d-1 \leq\left|w_{(1)}\right|+2\left|F\left(w_{(1)}\right)\right|+d-1 .
$$

By (5.3a), we have $\left|F\left(w_{(1)}\right)\right|=\left|w_{0}\right|$, hence

$$
\left|w_{(d)}\right| \leq\left|w_{(1)}\right|+2\left|w_{0}\right|+d-1 .
$$

Condition (5.3c) implies $\left|w_{(1)}\right| \leq|w|+2(h-1)+2\left|w_{h}\right|+\left|D\left(w_{(1)}\right)\right|$. Using the relation $|w| \geq\left|w_{0}\right|+h+\left|w_{h}\right|$, we obtain

$$
\left|w_{(d)}\right| \leq 3|w|+d+\left|D\left(w_{(1)}\right)\right|-3 .
$$


By construction, $d$ is the length of $u$. As (5.3b) implies $\left|D\left(w_{(1)}\right)\right| \leq 2$, we find

$$
\left|w_{(d)}\right| \leq 3|w|+|u|-1,
$$

which completes the case $p \leq n-2$ writing $w^{\prime}=w_{(d)}$.

Assume now $p=n-1$. Then the word $u$ is empty. Put $w=w^{\prime \prime} a_{q-1, n-1}$, and write $w^{\prime}=w^{\prime \prime} d_{q-1, n-1} d_{q-1, n-2}^{-1}$. The word $w^{\prime}$ is clearly an $a_{n-1, n}$-wall lent on $a_{q-1, n-1}$ and all complexity statements are satisfied. Moreover, for $q=n-1$ and $w \neq a_{n-2, n-1}$, Lemma 3.2(iii) implies that $w^{\prime \prime}$ ends with $a_{r, n-1}$ for some $r$, hence it is $\sigma_{n-2}$-positive. Then $w^{\prime}$ has the expected properties.

Finally, assume $p \neq n-1, q=n-1$ and $w \neq a_{n-2, n-1}$. Then $u$ is not empty. By hypothesis, the last letter of $w$ is $a_{n-2, n-1}$, which is not a barrier. Hence the word $w_{h}$ is not empty and its last letter $a_{n-2, n-1}$. Then (5.3d) implies that the wall $w_{(1)}$ is high. Hence, (5.5d) implies that the wall $w_{(k)}$ is high for every $d \geq k \geq 1$, and therefore $w^{\prime}$ is a high wall. By definition, $w^{\prime}$ can be expressed as $u d_{r, n-1} \widehat{w} d_{n-2, n-1}$. By construction, $u d_{r, n-1} \widehat{w}$ is a $\sigma_{n-2}$-positive word, so $w^{\prime}$ has all expected properties.

As for the time complexity upper bound, it follows from an easy bookkeeping argument using Lemmas 5.3 and 5.6, and the fact that the cost of one reversing step is $O(1)$.

Example 5.8. Let $w$ be the $a_{3,7}$-ladder $a_{4,6} a_{1,4} a_{2,6}$ and $u$ be the $a_{3,7}$-dangerous word $d_{5,6}^{-1} d_{3,6}^{-1} d_{3,6}^{-1}$. The reversing diagram for $u w$ is displayed in Figure 11 .

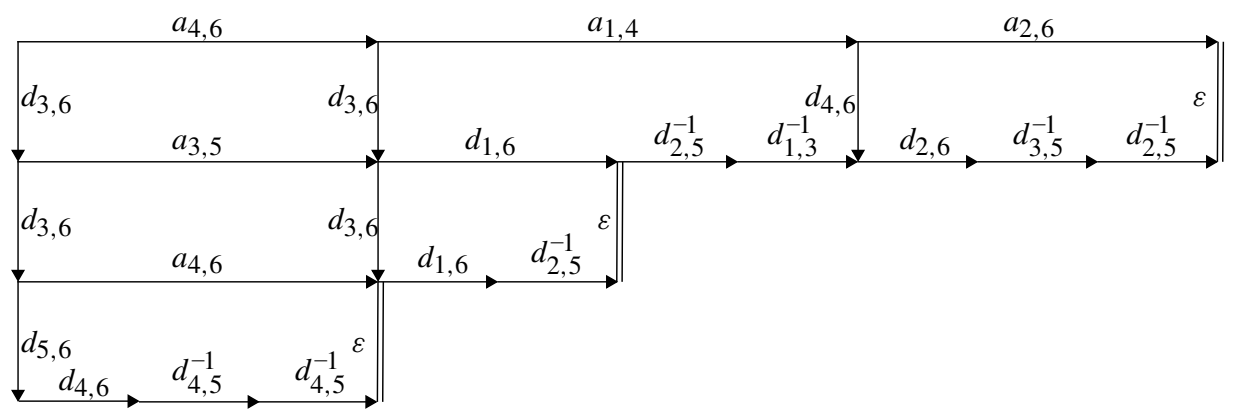

Fig. 11. Reversing $u w$ into a wall. Here $u$ is the $a_{3,7}$-dangerous word $d_{5,6}^{-1} d_{3,6}^{-1} d_{3,6}^{-1}$ and $w$ is the $a_{3,7}$-ladder $a_{4,6} a_{1,4} a_{2,6}$, which is lent on $a_{2,6}$. With the notation of Proposition 5.7, $w_{(1)}$ is the word $a_{3,5} d_{1,6} d_{2,5}^{-1} d_{1,3}^{-1} d_{2,6} d_{3,5}^{-1} d_{2,5}^{-1}$ : it can be read (from left to right) on the third row from the bottom. Then $w_{(2)}$ is the word $a_{4,6} d_{1,6} d_{2,5}^{-1} d_{2,5}^{-1} d_{1,3}^{-1} d_{2,6} d_{3,5}^{-1} d_{2,5}^{-1}$ : it can be read on the second row, continuing on the third row when the vertical $\varepsilon$-labeled edge is met. Finally $w_{(3)}$ is the word $d_{4,6} d_{4,5}^{-1} d_{4,5}^{-1} d_{1,6} d_{2,5}^{-1} d_{2,5}^{-1} d_{1,3}^{-1} d_{2,6} d_{3,5}^{-1} d_{2,5}^{-1}$ : it can be read on the bottom row, continued on the second row, and finally on the third row. The point is that we had three negative $d_{., 6}$-letters at first and, at each step, we got rid of one of them, ending with a word that contains negative $d_{.,}$, -letters for $q \leq 5$ only.

We conclude the section with one more technical result, which provides the precise basic step needed in the inductive definition of our final normal form $\underline{\mathrm{NF}}_{n}$. 
Lemma 5.9. Assume that $n \geq 3$, and

- $\left(w_{b}, \ldots, w_{1}\right)$ is the $\phi_{n}$-splitting of a normal word $w$, with $b \geq 3$,

- $u_{b}$ is a $w_{b}^{\#}$-dangerous word,

- $c$ is a number in $\{b, \ldots, 3\}$.

Then there exists

- a $\sigma_{n-1}$-nonnegative word $w^{\prime}$,

- $a w_{c}^{\#}$-dangerous word $u_{c}$,

both computable in time $O\left(\left|u_{b}\right||w|^{2}\right)$, that satisfy

$$
d_{1, n}^{-b+3} \phi_{n}^{b-1}\left(u_{b}\right) \phi_{n}^{b-2}\left(w_{b-1}\right) \ldots w_{1} \equiv w^{\prime} \cdot d_{1, n}^{-c+3} \phi_{n}^{c-1}\left(u_{c}\right) \phi_{n}^{b-2}\left(w_{c-1}\right) \ldots w_{1},
$$

with $\left|w^{\prime}\right| \leq 3\left|w_{b-1}\right|+\cdots+3\left|w_{c}\right|+\left|u_{b}\right|-\left|u_{c}\right|-b+c$ and $\left|u_{c}\right| \leq\left|u_{b}\right|+b$.

Proof. The idea is as follows: using induction on $k$ going from $b$ to $c+1$, we compute a $\sigma_{n-1}$-nonnegative word $w_{k-1}^{\prime}$ and a $w_{k}^{\#}$-dangerous word $u_{k-1}$ satisfying

$$
d_{1, n}^{-k+3} \phi_{n}^{k-1}\left(u_{k}\right) \phi_{n}^{k-2}\left(w_{k-1}\right) \equiv w_{k-1}^{\prime} d_{1, n}^{-k+4} \phi_{n}^{k-2}\left(u_{k-1}\right) .
$$

Then we define $w^{\prime}$ to be $w_{b-1}^{\prime} \ldots w_{c}^{\prime}$.

Let us go into details. First we construct the words $w_{k}^{\prime}$ and $u_{k}$. Fix $k$ in $\{b, \ldots, c+1\}$ and assume that $u_{k}$ is a $w_{k}^{\#}$-dangerous word. Corollary 3.10 guarantees that $w_{k-1}$ is a $\phi_{n}\left(w_{k}^{\#}\right)$-ladder lent on $w_{k-1}^{\#}$. Then, by Proposition 5.7, the word $\phi_{n}\left(u_{k}\right) w_{k-1}$ is equivalent to an $a_{t, n}$-wall $v_{k-1}$ lent on $w_{k-1}^{\#}$ for some $t$. By the definition of a wall, we have

$$
v_{k-1}=v_{k-1}^{\prime} d_{p-1, n-1} u_{k-1},
$$

where $v_{k-1}^{\prime}$ is a $\sigma_{n-2}$-nonnegative word, $u_{k-1}$ is a $w_{k-1}^{\#}$-dangerous word and $a_{p-1, n-1}$ is the last letter of $w_{k-1}$. Then we obtain

$$
d_{1, n}^{-k+3} \phi_{n}^{k-1}\left(u_{k}\right) \phi_{n}^{k-2}\left(w_{k-1}\right) \equiv d_{1, n}^{-k+3} \phi_{n}^{k-2}\left(v_{k-1}^{\prime} d_{p-1, n-1} u_{k-1}\right) .
$$

We push the power of $d_{1, n}^{-1}$ to the last word between $d_{p-1, n-1}$ and $u_{k-1}$ :

$$
d_{1, n}^{-k+3} \phi_{n}^{k-2}\left(v_{k-1}^{\prime} d_{p-1, n-1} u_{k-1}\right) \equiv \phi_{n}\left(v_{k-1}^{\prime} d_{p-1, n-1}\right) d_{1, n}^{-k+3} \phi_{n}^{k-2}\left(u_{k-1}\right)
$$

By relation (4.3a), we have $\phi_{n}\left(d_{p-1, n-1}\right) d_{1, n}^{-1} \equiv d_{1, p}^{-1}$. Eventually, we obtain

$$
d_{1, n}^{-k+3} \phi_{n}^{k-1}\left(u_{k}\right) \phi_{n}^{k-2}\left(w_{k-1}\right) \equiv \phi_{n}\left(v_{k-1}^{\prime}\right) d_{1, p}^{-1} d_{1, n}^{-k+4} \phi_{n}^{k-2}\left(u_{k-1}\right) .
$$

Writing $w_{k-1}^{\prime}=\phi_{n}\left(v_{k-1}^{\prime}\right) d_{1, p}^{-1}$, Relation (5.10) implies (5.9). By construction, $w_{k-1}^{\prime}$ is $\sigma_{n-1}$-nonnegative and $u_{k}$ is an $w_{k}^{\#}$-dangerous word.

Gathering the relations (5.9) for $k$ from $b$ to $c+1$, we obtain the relation (5.8) for $w^{\prime}=w_{b-1}^{\prime} \ldots w_{c}^{\prime}$.

By construction, $w_{k}^{\prime}$ is $\sigma_{n-1}$-nonnegative for each $k$, hence $w^{\prime}$ is $\sigma_{n-1}$-nonnegative as well. 
It remains to establish the complexity statements. For every $k$ in $\{b, \ldots, c+1\},(5.6 \mathrm{~b})$ implies $\left|v_{k-1}\right| \leq 3\left|w_{k-1}\right|+\left|u_{k}\right|-1$. Then, by the construction of $w_{k-1}^{\prime}$, we have $\left|w_{k-1}^{\prime}\right| \leq$ $3\left|w_{k-1}\right|+\left|u_{k}\right|-\left|u_{k-1}\right|-1$. We deduce

$$
\left|w^{\prime}\right|=\left|w_{b-1}^{\prime} \ldots w_{c}^{\prime}\right| \leq 3\left|w_{b-1} \ldots w_{c}\right|+\left|u_{b}\right|-\left|u_{c}\right|-b+c .
$$

For each $k$, (5.6a) implies $\left|u_{k}\right| \leq\left|u_{k+1}\right|+1$. Then we find $\left|u_{k}\right| \leq\left|u_{b}\right|+b-k$, hence $\left|u_{k}\right| \leq\left|u_{b}\right|+b$. By Proposition 5.7, computing $u_{k}$ and $v_{k}$ from $w_{k}$ and $u_{k+1}$ requires at most $O\left(\left|u_{k+1}\right||w|+1\right)$ steps. Therefore, computing $w^{\prime}$ and $u_{c}$ from $u_{b}$ and the $\phi_{n}$ splitting of $w$ requires at most $O\left(\left(\left|u_{b}\right|+b\right)|w|+b-c\right)$ steps. As $b \leq|w|+2$, we deduce that computing $w^{\prime}$ and $u_{c}$ from $u_{b}$ and the $\phi_{n}$-splitting of $w$ requires at most $O\left(\left|u_{b}\right||w|^{2}\right)$ steps.

\section{The main result}

We are now ready to establish Theorems 1 and 2 of the introduction. What we shall do is to construct, for each $n$-strand braid $\beta$, a certain $a d$-word $\operatorname{NF}_{n}(\beta)$ that represents $\beta$ and is $\sigma$-definite, i.e., a word on the letters $a_{p, q}$ and $d_{p, q}$ which, translated to the alphabet of $\sigma_{i}$, becomes either $\sigma$-positive or $\sigma$-negative.

The construction of $\mathrm{NF}_{n}(\beta)$ involves two steps. The first (easy) step, described in Section 6.1, consists in extending the rotating normal form of Section 2.2 to all of $B_{n}$ by appending convenient denominators. The process is based on the Garside structure of the monoid $B_{n}^{+*}$.

The second step starts from the rotating normal form, and is described in Section 6.2. The process splits into three cases according to the relative size of two parameters associated with $\beta$, namely the breadth of the numerator and the exponent of the denominator in the rotating normal form of $\beta$. The reversing machinery developed in Sections 4 and 5 is needed to treat the difficult case when the above two parameters are close to one another.

\subsection{The rotating normal form of an arbitrary braid}

As mentioned above, we first extend the rotating normal form, so far defined only for those braids that belong to the monoid $B_{n}^{+*}$, to all braids.

Proposition 6.1. Each braid $\beta$ admits a unique expression $d_{1, n}^{-t} w$ where $t$ is a nonnegative integer, $w$ is a (rotating) normal word, and the braid $\bar{w}$ is not left-divisible by $d_{1, n}$ unless $t$ is zero.

Proof. By Proposition 1.5, the monoid $B_{n}^{+*}$ is a Garside monoid with Garside element $\delta_{n}$, and the group $B_{n}$ is a group of fractions for the monoid $B_{n}^{+*}$. Hence, there exists a smallest integer $t$ such that $\delta_{n}^{t} \beta$ belongs to $B_{n}^{+*}$. If $t$ is positive, the minimality hypothesis implies that $\delta_{n}$ is not a left-divisor of $\delta_{n}^{t} \beta$. Taking for $w$ the rotating normal form of $\delta_{n}^{t} \beta$ gives a pair $(t, w)$ of the expected form-we recall that $d_{1, n} \equiv \delta_{n}$.

Assume that $\left(t^{\prime}, w^{\prime}\right)$ is another pair with the above properties. Then $\delta_{n}^{t^{\prime}} \beta$ belongs to $B_{n}^{+*}$, hence $t^{\prime} \geq t$. If we had $t^{\prime}>t$, the hypothesis $\delta_{n}^{-t} \bar{w}=\delta_{n}^{-t^{\prime}} \overline{w^{\prime}}$ would yield 
$\delta_{n}^{t^{\prime}-t} \bar{w}=\overline{w^{\prime}}$, implying that $\overline{w^{\prime}}$ is left-divisible by $\delta_{n}$, which contradicts $t^{\prime}>0$. Hence $t^{\prime}=t$, whence $w^{\prime}=w$ by uniqueness of the rotating normal form.

Definition 6.2. The $a d$-word $d_{1, n}^{-t} w$ involved in Proposition 6.1 is called the $n$-rotating normal form of the braid $\beta$. The number $t$ is called the $n$-depth of $\beta$; the number $t+|w|$, i.e., the length of the $a d$-word $d_{1, n}^{-t} w$, is called the $n$-length of $\beta$, denoted $|\beta|_{n}$; finally, for $n \geq 3$, the $n$-breadth of $w$ is called the $n$-breadth of $\beta$.

By definition, the rotating normal form of a braid is an $a d$-word, i.e., a word involving the letters $a_{p, q}$ and $d_{p, q}$ (actually the letter $d_{1, n}^{-1}$ only). The terminology is coherent since, for $\beta$ in $B_{n}^{+*}$, the rotating normal form as defined above coincides with the rotating normal form of Definition 2.11: indeed, $\beta$ belongs to $B_{n}^{+*}$ if and only if its $n$-depth is 0 .

Building on Proposition 2.13 and on the Garside structure of $B_{n}^{+*}$, we easily see that the rotating normal form of an arbitrary braid can be computed in quadratic time.

Lemma 6.3. For $n \geq 3$ and $1 \leq i \leq n-1$, let $\theta_{i, n}$ be the $a$-word $\phi_{n}^{i+1}\left(\delta_{n-1}\right)$. Then $\theta_{i, n}$ is equivalent to $\delta_{n} \sigma_{i}^{-1}$, and it has length $n-2$.

Proof. By Lemma 1.6, we have $\phi_{n}^{i+1}\left(a_{n-1, n}\right)=a_{i, i+1}$. We deduce $\phi_{n}^{i+1}\left(\delta_{n}\right) \equiv \phi_{n}^{i+1}\left(\delta_{n-1}\right) \sigma_{i}$ $=\theta_{i, n} \sigma_{i}$. As $\delta_{n}$ is invariant under $\phi_{n}$, we have $\delta_{n}=\theta_{i, n} \sigma_{i}$. The length of the $a$-word $\delta_{n-1}$ is $n-2$. As $\phi_{n}$ preserves the length of $a$-words, the length of $\theta_{i, n}$ is $n-2$.

We recall that, for $\beta$ a braid, $\|\beta\|_{\sigma}$ denotes the length of the shortest expression of $\beta$ in terms of the Artin generators $\sigma_{i}$.

Proposition 6.4. For each $n$-strand braid $\beta$, we have $|\beta|_{n} \leq(n-1)\|\beta\|_{\sigma}$. Moreover, if $\beta$ is specified by a word of length $\ell$, the rotating normal form of $\beta$ can be computed in time $O\left(\ell^{2}\right)$.

Proof. The case $n=2$ is trivial. Starting with a word on the alphabet $\left\{\sigma_{1}, \sigma_{1}^{-1}\right\}$, we freely reduce it to $\sigma_{1}^{k}$ by deleting the factors $\sigma_{1} \sigma_{1}^{-1}$ and $\sigma_{1}^{-1} \sigma_{1}$. The rotating normal form is $a_{1,2}^{k}$ in the case $k \geq 0$, and $d_{1,2}^{k}$ in the case $k<0$, and it is geodesic.

Assume now $n \geq 3$. Let $w$ be an $n$-strand braid word representing $\beta$. Then the rotating normal form of $\beta$ is obtained as follows:

- Replace each positive letter $\sigma_{i}$ in $w$ with $a_{i, i+1}$, so as to obtain

$$
u=w_{0} \sigma_{i_{1}}^{-1} w_{1} \ldots w_{c-1} \sigma_{i_{c}}^{-1} w_{c}
$$

- Put $v=\phi_{n}^{c}\left(w_{0}\right) \phi_{n}^{c-1}\left(\theta_{i_{1}, n} w_{1}\right) \ldots \phi_{n}\left(\theta_{i_{c-1}, n} w_{c-1}\right) \theta_{i_{c}, n} w_{c}$.

- Let $s$ be the maximal integer such that $\delta_{n}^{s}$ left-divides $\bar{v}$ in $B_{n}^{+*}$, and let $v^{\prime}$ be a positive $a$-word satisfying $v \equiv \delta_{n}^{s} v^{\prime}$.

- If $s \geq c$, put $t=0$ and $w^{\prime \prime}=\delta_{n}^{s-c} v^{\prime}$; otherwise put $t=c-s$ and $w^{\prime \prime}=v^{\prime}$.

- Let $w^{\prime}$ be the normal form of $\overline{w^{\prime \prime}}$. Then the rotating normal form of $\beta$ is $d_{1, n}^{-t} w^{\prime}$. 
Indeed, Lemma 6.3 and $\delta_{n} \equiv d_{1, n}$ imply

$$
w \equiv w_{0} d_{1, n}^{-1} \theta_{i_{1}, n} w_{1} \ldots d_{1, n}^{-1} \theta_{i_{c-1}, n} w_{c-1} d_{1, n}^{-1} \theta_{i_{c}, n} w_{c} .
$$

Pushing the letters $d_{1, n}^{-1}$ to the left, we obtain

$$
w \equiv d_{1, n}^{-c} \phi_{n}^{c}\left(w_{0}\right) \phi_{n}^{c-1}\left(\theta_{i_{1}, n} w_{1}\right) \ldots \phi_{n}\left(\theta_{i_{c-1}, n} w_{c-1}\right) \theta_{i_{c}, n} w_{c}=d_{1, n}^{-c} v .
$$

Using the relation $d_{1, n} \equiv \delta_{n}$ and the construction of $w^{\prime}$, we obtain $w \equiv d_{1, n}^{-t} w^{\prime}$, where $\overline{w^{\prime}}$ is not left-divisible by $d_{1, n}$ unless $t$ is zero.

As for the length, replacing $\sigma_{i_{k}}^{-1}$ by $d_{1, n}^{-1} \theta_{i_{k}}$ multiplies it by at most $n-1$. Applying the construction in the case when $w$ is a shortest expression of $\beta$ gives $|\beta|_{n} \leq(n-1)\|\beta\|_{\sigma}$.

As for the time complexity, $v$ is obtained in time $O(\ell)$, the integer $s$ is obtained in time $O\left(\ell^{2}\right)$ —see for instance [13]—and $w^{\prime}$ is obtained in time $O\left(\left|w^{\prime \prime}\right|^{2}\right)$ by Proposition 2.13. Hence, as $\left|w^{\prime \prime}\right| \leq \ell$, the rotating normal form of $\beta$ is obtained from the word $w$ in time $O\left(\ell^{2}\right)$.

Example 6.5. Consider $\beta=\sigma_{1} \sigma_{3}^{-2} \sigma_{2} \sigma_{3}$. We use the notation of Proposition 6.4. First, we write $u=w_{0} \sigma_{3}^{-1} w_{1} \sigma_{3}^{-1} w_{2}$ with $w_{0}=a_{1,2}, w_{1}=\varepsilon$ and $w_{2}=a_{2,3} a_{3,4}$. Then we have $\theta_{3,4}=\phi_{4}^{4}\left(\delta_{3}\right)=a_{1,2} a_{2,3}$, and we find

$$
v=\phi_{4}^{2}\left(w_{0}\right) \phi_{4}\left(\theta_{3,4} w_{1}\right) \theta_{3,4} w_{2}=a_{3,4} a_{2,3} a_{3,4} a_{1,2} a_{2,3} a_{2,3} a_{3,4} .
$$

The maximal power of $\delta_{4}$ that left-divides $\bar{v}$ is 1 and we have $v \equiv \delta_{4} a_{2,3} a_{1,2} a_{2,3} a_{2,4}$. So we find $s=1$ and $v^{\prime}=a_{2,3} a_{1,2} a_{2,3} a_{2,4}$. Here we have $c=2$ and $s=1$, hence we put $t=1$ and $w^{\prime \prime}=a_{2,3} a_{1,2} a_{2,3} a_{2,4}$. The rotating normal form $w^{\prime}$ of $\overline{w^{\prime \prime}}$ turns out to be $a_{1,2} a_{1,4} a_{2,3} a_{1,2}$. So, finally, the rotating normal form of $\beta$ is

$$
d_{1,4}^{-1} a_{1,2} a_{1,4} a_{2,3} a_{1,2} .
$$

Hence the 4-depth of $\beta$ is 1, its length is 5, and its 4-breadth is 4, since we saw in Example 2.15 that the 4-breadth of $a_{1,2} a_{1,4} a_{2,3} a_{1,2}$ is 4 : its $\phi_{4}$-splitting is $\left(a_{2,3}, a_{2,3}, 1, a_{2,3} a_{1,2}\right)$, a sequence of length 4 .

\subsection{The word $\mathrm{NF}_{n}(\beta)$ : the easy cases}

Starting from the rotating normal form, we shall now define for each braid $\beta$ a new distinguished expression $\mathrm{NF}_{n}(\beta)$ that is a $\sigma$-definite word. The word $\mathrm{NF}_{n}(\beta)$ will be constructed as a word on the letters $a_{p, q}$ and $d_{p, q}$. At the end, it will be obvious how to translate it into an ordinary braid word, i.e., a word on the letters $\sigma_{i}$.

The construction of $\mathrm{NF}_{n}(\beta)$ depends on the relative values of the $n$-depth and the $n$ breadth of $\beta$. The first case, which is easy, is when the $n$-depth of $\beta$ is 0 , i.e., $\beta$ belongs to $B_{n}^{+*}$, or it is $|\beta|_{n}$, i.e., $\beta$ is a negative power of $d_{1, n}$. Note that this case is the only possible one in the case of $B_{2}$.

Definition 6.6. Assume that $\beta$ is a braid of $B_{n}$ whose $n$-depth is 0 or $|\beta|_{n}$. Then we define $\mathrm{NF}_{n}(\beta)$ to be the $n$-rotating normal form of $\beta$. 
In this case, everything is clear.

Proposition 6.7. Under the hypotheses of Definition 6.6, the word $\mathrm{NF}_{n}(\beta)$ is a $\sigma$-definite expression of $\beta$, and its length is at most $|\beta|_{n}$. Moreover, if $\beta$ is specified by a $\sigma$-word of length $\ell$, the word $\mathrm{NF}_{n}(\beta)$ can be computed in time $O\left(\ell^{2}\right)$.

Proof. If $|\beta|_{n}$ is equal to 0 , then $\beta$ is the trivial braid 1 and its rotating normal form is the empty word. If $\beta$ is nontrivial and its $n$-depth is 0 , then the rotating normal form is a nonempty positive $a$-word, i.e., a $\sigma$-positive word. If $\beta$ is nontrivial and its $n$-depth $t$ equals $|\beta|_{n}$, then the rotating normal form of $\beta$ is $d_{1, n}^{-t}$, which is $\sigma_{n-1}$-negative. The complexity statements are clear from Proposition 6.4.

The second case, which is easy as well, is when the depth is large. We recall that, if $w$ is a normal word, then the $\phi_{n}$-splitting of $w$ is the sequence of normal words that represent the entries in the $\phi_{n}$-splitting of the braid represented by $w$.

Definition 6.8. Assume that $\beta$ is a nontrivial braid of $B_{n}$ with $n \geq 3$ whose $n$-depth $t$ satisfies $t \neq 0$ and $t>b-2$, where $b$ is the $n$-breadth of $\beta$. Let $d_{1, n}^{-t} w$ be the rotating normal form of $\beta$ and $\left(w_{b}, \ldots, w_{1}\right)$ be the splitting of $w$. Then we put

$$
\mathrm{NF}_{n}(\beta)=d_{1, n}^{-t+b-1} \cdot w_{b} d_{1, n}^{-1} \cdot \ldots \cdot w_{2} d_{1, n}^{-1} \cdot w_{1} .
$$

Proposition 6.9. Under the hypotheses of Definition 6.8, the word $\mathrm{NF}_{n}(\beta)$ is a $\sigma$-negative expression of $\beta$, and its length is at most $|\beta|_{n}$. Moreover, if $\beta$ is specified by a $\sigma$-word of length $\ell$, the word $\mathrm{NF}_{n}(\beta)$ can be computed in time $O\left(\ell^{2}\right)$.

Proof. First, we claim that $\mathrm{NF}_{n}(\beta)$ is an expression of $\beta$. Let $d_{1, n}^{-t} w$ be the rotating normal form of $\beta$ and $\left(w_{b}, \ldots, w_{1}\right)$ be the $\phi_{n}$-splitting of $w$. We have

$$
d_{1, n}^{-t} w=d_{1, n}^{-t} \cdot \phi_{n}^{b-1}\left(w_{b}\right) \cdot \ldots \cdot \phi_{n}\left(w_{2}\right) \cdot w_{1} .
$$

Pushing $b-1$ powers of $d_{1, n}$ to the right in (6.1) and dispatching them between the factors $w_{k}$, we find

$$
\begin{aligned}
d_{1, n}^{-t} w & =d_{1, n}^{-t} \cdot \phi_{n}^{b-1}\left(w_{b}\right) \cdot \ldots \cdot \phi_{n}\left(w_{2}\right) \cdot w_{1} \\
& =d_{1, n}^{-t+b-1} \cdot d_{1, n}^{-b+1} \cdot \phi_{n}^{b-1}\left(w_{b}\right) \cdot \ldots \cdot \phi_{n}\left(w_{2}\right) \cdot w_{1} \\
& \equiv d_{1, n}^{-t+b-1} \cdot w_{b} \cdot d_{1, n}^{-1} \cdot d_{1, n}^{-b+2} \cdot \ldots \cdot \phi_{n}\left(w_{2}\right) \cdot w_{1} \\
& \equiv \cdots \equiv d_{1, n}^{-t+b-1} \cdot w_{b} \cdot d_{1, n}^{-1} \cdot \ldots \cdot w_{2} \cdot d_{1, n}^{-1} \cdot w_{1}=\operatorname{NF}_{n}(\beta) .
\end{aligned}
$$

Next, $t$ being the $n$-depth of $\beta$, exactly $t$ powers of $d_{1, n}^{-1}$ occur in $\mathrm{NF}_{n}(\beta)$. Hence, as $t$ is not 0 , at least one $d_{1, n}^{-1}$ appears in $\mathrm{NF}_{n}(\beta)$. By construction, the intermediate words $w_{k}$ contain no letter $a_{p, n}$. Therefore, the word $\operatorname{NF}_{n}(\beta)$ is $\sigma_{n-1}$-negative.

As for the length, we find

$$
\begin{aligned}
\left|\mathrm{NF}_{n}(\beta)\right| & =t-b+1+\left|w_{b}\right|+1+\cdots+\left|w_{2}\right|+1+\left|w_{1}\right| \\
& =t-b+1+|w|+b-1=\left|d_{1, n}^{-t} w^{\prime}\right|=|\beta|_{n} .
\end{aligned}
$$


Finally, assume that $\beta$ is specified by a word of length $\ell$. Then, by Proposition 6.4, we can compute the rotating normal form of $\beta$ in at most $O\left(\ell^{2}\right)$ steps. By Lemma 2.14, computing the $\phi_{n}$-splitting of $w$ can be done in $O(|w|)$ steps. Hence, $\operatorname{NF}_{n}(\beta)$ can be computed in time $O\left(\ell^{2}\right)$.

\subsection{The word $\mathrm{NF}_{n}(\beta)$ : the difficult case}

There remains the case of a braid $\beta$ whose $n$-depth $t$ satisfies $t \neq 0$ and $t \leq b-2$, where $b$ is the $n$-breadth of $\beta$ : this is the difficult case. In this case, it is impossible to directly predict whether $\beta$ has a $\sigma_{n-1}$-positive or a $\sigma_{n-1}$-neutral word (i.e., one in which neither $\sigma_{n-1}$ nor $\sigma_{n-1}^{-1}$ occurs), and this is the point where we shall use the ladder and reversing machinery developed in Sections 3, 4 and 5.

Definition 6.10. Assume that $\beta$ is a nontrivial braid of $B_{n}$ with $n \geq 3$ whose $n$-depth $t$ satisfies $t \neq 0$ and $t \leq b-2$, where $b$ is the $n$-breadth of $\beta$. Let $\bar{d}_{1, n}^{-t} w$ be the rotating normal form of $\beta$, and $\left(w_{b}, \ldots, w_{1}\right)$ be the $\phi_{n}$-splitting of $w$. Write $w_{t+2}=w_{t+2}^{\prime} a_{p-1, n-1}$. Put

$$
v=\phi_{n}^{b-1-t}\left(w_{b}\right) \ldots \phi_{n}^{2}\left(w_{t+3}\right) \phi_{n}\left(w_{t+2}^{\prime}\right) d_{1, p}^{-1}, \quad u_{t+2}=d_{p-1, n-2}^{-1} .
$$

Case 1: $w_{2} \neq \varepsilon$. Then we put

$$
\mathrm{NF}_{n}(\beta)=v w^{\prime \prime} \phi_{n}\left(w_{2}^{\prime}\right) w_{1}
$$

where $w^{\prime \prime}$ and $u_{3}$ are the words produced by Lemma 5.9 applied to the sequence $\left(w_{t+2}, \ldots, w_{1}\right)$, the word $u_{t+2}$ and the integer 3 , and where $w_{2}^{\prime}$ is the word given by Proposition 5.7 applied to the words $w_{2}$ and $\phi_{n}\left(u_{3}\right)$.

Case 2: $w_{2}=\varepsilon, w_{3}=\cdots=w_{k-1}=a_{n-2, n-1}$ and $w_{k} \neq a_{n-2, n-1}$ for some $k \leq t+1$. Then we put

$$
\mathrm{NF}_{n}(\beta)=v w^{\prime \prime} \phi_{n}\left(w_{k}^{\prime}\right) d_{1, n-1}^{-k+2} w_{1},
$$

where $w^{\prime \prime}$ and $u_{k+1}$ are the words given by Lemma 5.9 applied to the sequence $\left(w_{t+2}, \ldots, w_{1}\right)$, the word $u_{t+2}$ and the integer $k+1$, and where $w_{k}^{\prime} a_{n-2, n-1}$ is the word produced by Proposition 5.7 applied to the words $w_{k}$ and $\phi_{n}\left(u_{k+1}\right)$.

Case 3: $w_{2}=\varepsilon, w_{3}=\cdots=w_{t+1}=a_{n-2, n-1}$ and $v \neq d_{1, n-1}^{-1}$. Then we put

$$
\mathrm{NF}_{n}(\beta)=v d_{1, n-1}^{-t+1} w_{1}
$$

Case 4: $w_{2}=\varepsilon, w_{3}=\cdots=w_{t+1}=a_{n-2, n-1}$ and $v=d_{1, n-1}^{-1}$. Then we put

$$
\mathrm{NF}_{n}(\beta)=\mathrm{NF}_{n-1}\left(\delta_{n-1}^{-t} \bar{w}_{1}\right) \text {. }
$$

Proposition 6.11. Under the hypotheses of Definition 6.10, the word $\mathrm{NF}_{n}(\beta)$ is a $\sigma$ definite expression of $\beta$, and its length is at most $3|\beta|_{n}$. Moreover, if $\beta$ is specified by $a$ $\sigma$-word of length $\ell$, the word $\mathrm{NF}_{n}(\beta)$ can be computed in time $O\left(\ell^{2}\right)$. 
Proof. We use the notation of Definition 6.10. First, we claim that the following equivalence holds:

$$
d_{1, n}^{-t} w \equiv v d_{1, n}^{-t+1} \phi_{n}^{t+1}\left(u_{t+2}\right) \phi_{n}^{t}\left(w_{t+1}\right) \ldots \phi_{n}\left(w_{2}\right) w_{1} .
$$

Indeed, as the sequence $\left(w_{b}, \ldots, w_{1}\right)$ is the $\phi_{n}$-splitting of $w$, we have

$$
d_{1, n}^{-t} w=d_{1, n}^{-t} \phi_{n}^{b-1}\left(w_{b}\right) \ldots \phi_{n}^{t+1}\left(w_{t+2}\right) \ldots \phi_{n}\left(w_{2}\right) w_{1} .
$$

By construction, $w_{t+2}$ is $w_{t+2}^{\prime} a_{p-1, n-1}$. By (4.1), we have $a_{p-1, n-1} \equiv d_{p-1, n-1} u_{t+2}$, hence $w_{t+2} \equiv w_{t+2}^{\prime} d_{p-1, n-1} u_{t+2}$. Then the word $d_{1, n}^{-t} w$ is equivalent to

$$
d_{1, n}^{-t} \phi_{n}^{b-1}\left(w_{b}\right) \ldots \phi_{n}^{t+1}\left(w_{t+2}^{\prime} d_{p-1, n-1}\right) \phi_{n}^{t+1}\left(u_{t+2}\right) \phi_{n}^{t}\left(w_{t+1}\right) \ldots \phi_{n}\left(w_{2}\right) w_{1} .
$$

We push the factor $d_{1, n}^{-t}$ appearing in (6.4) to the right, until it arrives at the left of the factor $\phi_{n}^{t+1}\left(u_{t+2}\right)$. In this way, we obtain

$$
d_{1, n}^{-t} w \equiv \phi_{n}^{b-t-1}\left(w_{b}\right) \ldots \phi_{n}\left(w_{t+2}^{\prime} d_{p-1, n-1}\right) d_{1, n}^{-t} \phi_{n}^{t+1}\left(u_{t+2}\right) \phi_{n}^{t}\left(w_{t+1}\right) \ldots \phi_{n}\left(w_{2}\right) w_{1} \text {. }
$$

Relations (4.3a) and (4.3b) imply $\phi_{n}\left(d_{p-1, n-1}\right) d_{1, n}^{-1} \equiv d_{1, p}^{-1}$. Inserting the latter value in the relation above, we obtain (6.2), as expected.

Next, by construction, the word $v$ is $\sigma_{n-1}$-nonnegative, and its length satisfies

$$
|v|=\left|w_{b}\right|+\cdots+\left|w_{t+2}\right|
$$

To go further, we consider the four cases of Definition 6.10 separately. In the first three cases, we shall show that $\mathrm{NF}_{n}(\beta)$ is $\sigma_{n-1}$-positive; in the fourth case, we shall show that $\mathrm{NF}_{n}(\beta)$ is $\sigma$-definite using induction on $n$ and possibly Propositions 6.7 and 6.9.

Case 1. First, $\mathrm{NF}_{n}(\beta)$ is equivalent to $d_{1, n}^{-t} w$. Indeed, Lemma 5.9 implies

$$
d_{1, n}^{-t} w \equiv v w^{\prime \prime} \phi_{n}^{2}\left(u_{3}\right) \phi_{n}\left(w_{2}\right) w_{1},
$$

while Proposition 5.7 implies $\phi_{n}\left(u_{3}\right) w_{2} \equiv w_{2}^{\prime}$. We deduce

$$
d_{1, n}^{-t} w \equiv v w^{\prime \prime} \phi_{n}\left(w_{2}^{\prime}\right) w_{1}=\mathrm{NF}_{n}(\beta)
$$

Next, by construction, $w_{2}^{\prime}$ is a wall lent on $w_{2}^{\#}$, hence, by definition, it is $\sigma_{n-2}$-positive. So $\phi_{n}\left(w_{2}^{\prime}\right)$ is $\sigma_{n-1}$-positive. As $v, w^{\prime \prime}$ and $w_{1}$ are $\sigma_{n-1}$-nonnegative, $\operatorname{NF}_{n}(\beta)$ is $\sigma_{n-1}$ positive.

As for the length, Lemma 5.9 and Proposition 5.7 imply

$$
\left|w^{\prime \prime}\right| \leq 3\left|w_{t+1}\right|+\cdots+3\left|w_{3}\right|-\left|u_{3}\right|-t+2, \quad\left|w_{2}^{\prime}\right| \leq 3\left|w_{2}\right|+\left|u_{3}\right|-1 .
$$

Merging these values with (6.5), and $t>0$, we deduce $\left|\mathrm{NF}_{n}(\beta)\right| \leq 3|w|$.

Case 2. First, we observe that the last letter of $w_{k}$ must be $a_{n-2, n-1}$ : this follows from Corollary 3.11 since, by the construction of $k$, the word $w_{k-1}$ is either $\varepsilon$ or $a_{n-2, n-1}$. 
Now, we check that $\operatorname{NF}_{n}(\beta)$ is equivalent to $d_{1, n}^{-t} w$. By Lemma 5.9, we have

$$
d_{1, n}^{-t} w \equiv v w^{\prime \prime} d_{1, n}^{-k+2} \phi_{n}^{k}\left(u_{k+1}\right) \phi_{n}^{k-1}\left(w_{k}\right) \phi_{n}^{k-2}\left(a_{n-2, n-1}\right) \ldots \phi_{n}^{2}\left(a_{n-2, n-1}\right) w_{1} .
$$

By Proposition 5.7, $\phi_{n}\left(u_{k+1}\right) w_{k}$ is equivalent to a wall $\tilde{w}_{k}$ lent on $w_{k}^{\#}=a_{n-2, n-1}$. We define $w_{k}^{\prime}$ by setting $\tilde{w}_{k}=w_{k}^{\prime} a_{n-2, n-1}$. Then

$$
d_{1, n}^{-t} w \equiv v w^{\prime \prime} d_{1, n}^{-k+2} \phi_{n}^{k-1}\left(w_{k}^{\prime}\right) \phi_{n}^{k-1}\left(a_{n-2, n-1}\right) \ldots \phi_{n}^{2}\left(a_{n-2, n-1}\right) w_{1} .
$$

Pushing the negative powers of $d_{1, n}$ appearing in (6.6) to the right and dispatching them between the $\phi_{n}\left(a_{n-2, n-1}\right)$, we find

$$
\begin{aligned}
d_{1, n}^{-t} w & \equiv v w^{\prime \prime} \phi_{n}\left(w_{k}^{\prime}\right) d_{1, n}^{-k+2} \phi_{n}^{k-1}\left(a_{n-2, n-1}\right) \ldots \phi_{n}^{2}\left(a_{n-2, n-1}\right) w_{1} \\
& \equiv v w^{\prime \prime} \phi_{n}\left(w_{k}^{\prime}\right) \phi_{n}\left(a_{n-2, n-1}\right) d_{1, n}^{-1} d_{1, n}^{-k+3} \ldots \phi_{n}^{2}\left(a_{n-2, n-1}\right) w_{1} \\
& \equiv \cdots \equiv v w^{\prime \prime} \phi_{n}\left(w_{k}^{\prime}\right) \phi_{n}\left(a_{n-2, n-1}\right) d_{1, n}^{-1} \ldots \phi_{n}\left(a_{n-2, n-1}\right) d_{1, n}^{-1} w_{1} .
\end{aligned}
$$

Then $\phi_{n}\left(a_{n-2, n-1}\right) d_{1, n}^{-1} \equiv d_{1, n-1}^{-1}$ implies

$$
d_{1, n}^{-t} w \equiv v w^{\prime \prime} \phi_{n}\left(w_{k}^{\prime}\right) d_{1, n-1}^{-k+2} w_{1}=\mathrm{NF}_{n}(\beta) .
$$

Next, by the construction of $w_{k}^{\prime}$ and the last statement of Proposition 5.7, the word $w_{k}^{\prime}$ is $\sigma_{n-2}$-positive. So $\phi_{n}\left(w_{k}^{\prime}\right)$ is $\sigma_{n-1}$-positive. As $v, w^{\prime \prime}$, and $d_{1, n-1}^{-k+2} w_{1}$, are $\sigma_{n-1}$-nonnegative, the word $\mathrm{NF}_{n}(\beta)$ is $\sigma_{n-1}$-positive.

As for the length, Lemma 5.9 and Proposition 5.7 imply

$$
\left|w^{\prime \prime}\right| \leq 3\left|w_{t+1}\right|+\cdots+3\left|w_{3}\right|-\left|u_{k+1}\right|-t+2, \quad\left|w_{k}^{\prime} a_{n-2, n-1}\right| \leq 3\left|w_{k}\right|+\left|u_{k+1}\right|-1 .
$$

Merging these values with (6.5) and the hypothesis $t>0$, we find $\left|\mathrm{NF}_{n}(\beta)\right| \leq 3|w|$.

Case 3. As above, we observe that the last letter of $w_{t+2}$ is $a_{n-2, n-1}$, which follows from Corollary 3.11, since $w_{t+1}$ is either 1 or $a_{n-2, n-1}$.

Then we check that $\operatorname{NF}_{n}(\beta)$ is equivalent to $d_{1, n}^{-t} w$. As the last letter of $w_{t+2}$ is $a_{n-2, n-1}$, the word $u_{t+2}$ is empty. Then we find

$$
d_{1, n}^{-t} w \equiv v d_{1, n}^{-t+1} \phi_{n}^{t}\left(a_{n-2, n-1}\right) \ldots \phi_{n}^{2}\left(a_{n-2, n-1}\right) \phi_{n}(\varepsilon) w_{1} .
$$

Pushing again the negative powers of $d_{1, n}$ of (6.7) to the right and dispatching them between the $\phi_{n}\left(a_{n-2, n-1}\right)$, we find

$$
\begin{aligned}
d_{1, n}^{-t} w & \equiv v \phi_{n}\left(a_{n-2, n-1}\right) d_{1, n}^{-1} d_{1, n}^{-t+2} \phi_{n}^{t-1}\left(a_{n-2, n-1}\right) \ldots \phi_{n}^{2}\left(a_{n-2, n-1}\right) w_{1} \\
& \equiv \cdots \equiv v \phi_{n}\left(a_{n-2, n-1}\right) d_{1, n}^{-1} \ldots \phi_{n}\left(a_{n-2, n-1}\right) d_{1, n}^{-1} w_{1} .
\end{aligned}
$$

Then $\phi_{n}\left(a_{n-2, n-1}\right) d_{1, n}^{-1} \equiv d_{1, n-1}^{-1}$ implies

$$
d_{1, n}^{-t} w \equiv v d_{1, n-1}^{-t+1} w_{1}=\mathrm{NF}_{n}(\beta) .
$$


Next, we check that $\operatorname{NF}_{n}(\beta)$ is $\sigma_{n-1}$-positive. As $w_{t+2}^{\#}=a_{n-2, n-1}$, we have

$$
v=\phi_{n}^{b-1-t}\left(w_{b}\right) \ldots \phi_{n}^{2}\left(w_{t+3}\right) \phi_{n}\left(w_{t+2}^{\prime}\right) d_{1, n-1}^{-1}
$$

By Lemma 3.2(iii), if the word $w_{t+2}^{\prime}$ is not empty, it ends with a letter of the form $a_{., n-1}$, hence $v$ is $\sigma_{n-1}$-positive. Assume that $w_{t+2}^{\prime}$ is empty and $t \leq b-3$. As the word $w_{t+2}$ is $a_{n-2, n-1}$, Corollary 3.11 implies that $w_{t+3}$ ends with $a_{n-2, n-1}$. Then $v$ ends with $\phi_{n}^{2}\left(a_{n-2, n-1}\right) d_{1, n-1}^{-1}$, which is $a_{1, n} d_{1, n-1}^{-1}$, hence $v$ is $\sigma_{n-1}$-positive.

Relation (6.5) directly implies $\left|\mathrm{NF}_{n}(\beta)\right|=|w|$.

Case 4. By construction, we have $v=d_{1, n-1}^{-1}$. The same analysis as in Case 3 gives $t=b-2$ and

$$
d_{1, n}^{-t} w \equiv d_{1, n-1}^{-t} w_{1}
$$

The induction hypothesis together with Propositions 6.7 and 6.9 gives $d_{1, n-1}^{-t} w_{1} \equiv$ $\mathrm{NF}_{n-1}\left(\delta_{n-1}^{-t} \overline{w_{1}}\right)$, hence $d_{1, n}^{-t} w \equiv \mathrm{NF}_{n}(\beta)$ by definition.

Always by the induction hypothesis and Propositions 6.7 and 6.9, we have

$$
\left|\mathrm{NF}_{n}(\beta)\right|=\left|\mathrm{NF}_{n-1}\left(\delta_{n-1}^{-t} \overline{w_{1}}\right)\right| \leq 3\left|\delta_{n-1}^{-t} \overline{w_{1}}\right|_{n-1} .
$$

By definition, we have $|\beta|_{n}=t+\left|w_{b}\right|+\cdots+\left|w_{1}\right|$ and $\left|\delta_{n-1}^{-t} \overline{w_{1}}\right|_{n-1} \leq t+\left|w_{1}\right|$, hence $\left|\delta_{n-1}^{-t} \overline{w_{1}}\right|_{n-1} \leq|\beta|_{n}$. Thus, we obtain $\left|\mathrm{NF}_{n}(\beta)\right| \leq 3|\beta|_{n}$.

So all cases have been considered, and it only remains to analyze the time complexity. By Proposition 6.4 and Lemma 2.14, the rotating normal form of $\beta$ and the $\phi_{n}$-splitting of $w$ can be computed in time $O\left(\ell^{2}\right)$. Then, in Cases 1 and 2, Lemma 5.9 is used once for $\left(w_{t+2}, \ldots, w_{1}\right)$ and $u_{t+2}$, with a cost $O\left(\ell^{2}\right)$. In addition, Proposition 5.7 is used at most once with $\phi_{n}\left(u_{k+1}\right)$ and $w_{k}(k=2$ for Case 1$)$, with a cost at most $O\left(\max \left(1,\left|u_{k+1}\right| \ell\right)\right)$. Lemma 5.9 guarantees $\left|u_{k+1}\right| \leq\left|u_{k+1}\right|+t+1-c$, i.e., $\left|u_{t+2}\right| \leq t$. So the total cost entailed by Proposition 5.7 is at most $O\left(\ell^{2}\right)$. The other computations in Cases 1, 2, and 3 require at most $O(\ell)$ steps, and therefore the total cost of the computation of $\mathrm{NF}_{n}(\beta)$ is $O\left(\ell^{2}\right)$ in Cases 1, 2 and 3. The result is similar for Case 4, using the induction hypothesis, and possibly Propositions 6.7 and 6.9.

\subsection{Putting things together}

Using the $\sigma$-definite words $\operatorname{NF}_{n}(\beta)$ constructed in Sections 6.2 and 6.3, we are now ready to establish Theorems 1 and 2 of the introduction. As a preliminary remark, we observe that the words $\operatorname{NF}_{n}(\beta)$ do not really depend on the index $n$.

Lemma 6.12. If $\beta$ belongs to $B_{n-1}$, the words $\mathrm{NF}_{n}(\beta)$ and $\mathrm{NF}_{n-1}(\beta)$ coincide.

Proof. An easy verification shows that, if $\beta$ belongs to $B_{n-1}$ and has $n$-depth $t$, then either $t=0$ (if $\beta$ belongs to $B_{n-1}^{+*}$ ), or we are in Case 4 of Definition 6.10. In both cases, the definition of $\mathrm{NF}_{n}(\beta)$ implies $\mathrm{NF}_{n}(\beta)=\mathrm{NF}_{n-1}(\beta)$.

So, from now on, we can skip the subscript $n$ and write $\operatorname{NF}(\beta)$ without ambiguity. The main result, of which Theorems 1 and 2 are easy consequences, is as follows. We recall that, for $\beta$ a braid, $\|\beta\|_{\sigma}$ denotes the length of the shortest expression of $\beta$ in terms of the Artin generators $\sigma_{i}$. 
Theorem 6.13. For each $n$-strand braid $\beta$, the ad-word $\mathrm{NF}(\beta)$ is a $\sigma$-definite expression of $\beta$, and its length is at most $3(n-1)\|\beta\|_{\sigma}$. Moreover, if $\beta$ is specified by a $\sigma$-word of length $\ell$, the word $\mathrm{NF}(\beta)$ can be computed in time $O\left(\ell^{2}\right)$.

Proof. Everything is obvious in the case $n=2$, so we assume $n \geq 3$. Applying Proposition 6.1, and, according to the case, Proposition 6.7, 6.9, or 6.11, the word $\mathrm{NF}(\beta)$ is, in any case, a $\sigma$-definite expression of $\beta$, and its length is at most $3|\beta|_{n}$. On the other hand, Proposition 6.4 implies $|\beta|_{n} \leq(n-1)\|\beta\|_{\sigma}$, so we deduce the expected upper bound

$$
|\operatorname{NF}(\beta)| \leq 3(n-1)\|\beta\|_{\sigma} .
$$

Finally, gathering the complexity analysis of Propositions 6.4, 6.7, 6.9, and 6.11 shows that, in all cases, $\operatorname{NF}(\beta)$ can be computed in $O\left(\ell^{2}\right)$ steps when $\beta$ is specified by an initial word of length $\ell$.

As promised, we can now deduce Theorems 1 and 2 in a few words.

Proof of Theorem 1. Let $\underline{\mathrm{NF}}(\beta)$ be the translation of the $a d$-word $\mathrm{NF}(\beta)$ into a $\sigma$-word. The formulas of (4.2) show that the translation of a letter $a_{p, q}$ or $d_{p, q}$ with $q \leq n$ has length at most $2 n-3$. So (6.8) implies $|\underline{\mathrm{NF}}(\beta)| \leq 6(n-1)^{2}\|\beta\|_{\sigma}$.

Proof of Theorem 2. Translating $\mathrm{NF}(\beta)$ into $\underline{\mathrm{NF}}(\beta)$ has a linear time cost, so the quadratic upper bound for the computation of $\operatorname{NF}(\beta)$ established in Theorem 6.13 immediately gives a quadratic upper bound for the computation of $\underline{\mathrm{NF}}(\beta)$.

Let us now give a concrete example of the previous constructions.

Example 6.14. We consider the braid $\beta=\sigma_{1} \sigma_{3}^{-2} \sigma_{2} \sigma_{3}$ of Example 6.5 again. We saw above that its rotating normal form is the $a d$-word

$$
d_{1,4}^{-1} a_{1,2} a_{1,4} a_{2,3} a_{1,2}
$$

We saw in Example 2.15 that the $\phi_{4}$-splitting of $a_{1,2} a_{1,4} a_{2,3} a_{1,2}$ is $\left(w_{4}, \ldots, w_{1}\right)$, with

$$
w_{4}=a_{2,3}, \quad w_{3}=a_{2,3}, \quad w_{2}=\varepsilon, \quad \text { and } \quad w_{1}=a_{2,3} a_{1,2} .
$$

So the 4-depth of $\beta$ is 1 , whereas its 4 -breadth is 4 . As $1 \leq 4-2$, we are in the difficult case. With the notation of Definition 6.10, we have $t=1$ and $w_{3}=\varepsilon \cdot a_{2,3}$, so we first put $w_{3}^{\prime}=\varepsilon, p=3, v=\phi_{4}^{2}\left(w_{4}\right) \phi_{4}\left(w_{3}^{\prime}\right) d_{1, p}^{-1}$, and $u_{3}=d_{p-1,2}^{-1}$, i.e., in the current case, $v=a_{1,4} d_{1,3}^{-1}$ and $u_{3}=\varepsilon$. Then, as $w_{2}=\varepsilon, w_{3}=a_{2,3}$ and $v \neq d_{1,3}^{-1}$, we are in Case 3 of Definition 6.10. According to the latter, we define $\operatorname{NF}(\beta)=v d_{1,3}^{0} w_{1}$, i.e., $\operatorname{NF}(\beta)=a_{1,4} d_{1,3}^{-1} a_{2,3} a_{1,2}$. This $a d$-word is $\sigma_{3}$-positive: indeed, its $\sigma$-translation is the $\sigma$-word

$$
\underline{\mathrm{NF}}(\beta)=\sigma_{1} \sigma_{2} \sigma_{3} \sigma_{2}^{-1} \sigma_{1}^{-1} \sigma_{2}^{-1} \sigma_{1}^{-1} \sigma_{2} \sigma_{1}
$$

which contains one $\sigma_{3}$, but no $\sigma_{3}^{-1}$, and no $\sigma_{i}^{ \pm 1}$ with $i \geq 4$. 
In the very simple case of Example 6.14, the reversing machinery is not used (and directly guessing a $\sigma$-definite word equivalent to the initial word would have been easy). However, much more complicated phenomena may occur in general, in particular when the braid index reaches 5, which is the smallest value for which there exist ladders with more than one bar. All situations considered in Definition 6.10 may occur when the length and the braid index increase, and explicit examples can easily be found using a computer. The examples witnessing really complicated behaviors, typically requiring more than one reversing step, involve words that are too long to be given here. However their existence confirms the amazing intricacy of the braid relations.

As a consequence, we deduce that the complexity of the Dehornoy order of braids is at most quadratic.

Acknowledgments. The author thanks P. Dehornoy for his help in writing this paper.

\section{References}

[1] Bessis, D.: The dual braid monoid. Ann. Sci. École Norm. Sup. (4) 36, 647-683 (2003) Zbl 1064.20039 MR 2032983

[2] Bessis, D., Digne, F., Michel, J.: Springer theory in braid groups and the Birman-Ko-Lee monoid. Pacific J. Math. 205, 287-309 (2002) Zbl 1056.20023 MR 1922736

[3] Birman, J., Ko, K. H., Lee, S. J.: A new approach to the word and conjugacy problems in the braid groups. Adv. Math. 139, 322-353 (1998) Zbl 0937.20016 MR 1654165

[4] Bressaud, X.: A normal form for braids. J. Knot Theory Ramif. 17, 697-732 (2008) Zbl 1158.20019 MR 2427952

[5] Burckel, S.: The wellordering on positive braids. J. Pure Appl. Algebra 120, 1-17 (1997) Zbl 0958.20032 MR 1466094

[6] Dehornoy, P.: Braid groups and left distributive operations. Trans. Amer. Math. Soc. 345, 115-150 (1994) Zbl 0837.20048 MR 1214782

[7] Dehornoy, P.: A fast method for comparing braids. Adv. Math. 125, 200-235 (1997) Zbl 0882.20021 MR 1434111

[8] Dehornoy, P.: Groupes de Garside. Ann. Sci. École Norm. Sup. (4) 35, 267-306 (2002) Zbl 1017.20031 MR 1914933

[9] Dehornoy, P.: Complete positive group presentations. J. Algebra 268, 156-197 (2003) Zbl 1067.20035 MR 2004483

[10] Dehornoy, P.: Alternating normal forms for braids and locally Garside monoids. J. Pure Appl. Algebra 212, 2413-2439 (2008) Zbl 1191.20033 MR 2440256

[11] Dehornoy, P., Dynnikov, I., Rolfsen, D., Wiest, B.: Ordering Braids. Math. Surveys Monogr. 148, Amer. Math. Soc. (2002) Zbl 1163.20024 MR 2463428

[12] Dynnikov, I., Wiest, B.: On the complexity of braids. J. Eur. Math. Soc. 9, 801-840 (2007) Zbl 1187.20045 MR 2341833

[13] Epstein, D., Cannon, J., Holt, D., Levy, S., Paterson, M., Thurston, W.: Word Processing in Groups. Jones \& Bartlett (1992) Zbl 0764.20017 MR 1161694

[14] Fenn, R., Greene, M. T., Rolfsen, D., Rourke, C., Wiest, B.: Ordering the braid groups. Pacific J. Math. 191, 49-74 (1999) Zbl 1009.20042 MR 1725462

[15] Fromentin, J.: A well-ordering of dual braid monoids. C. R. Math. Acad. Sci. Paris 346, 729734 (2008) Zbl 1146.20313 MR 2427071 
[16] Garside, F. A.: The braid group and other groups. Quart. J. Math. Oxford Ser. 20, 235-254 (1969) Zbl 0194.03303 MR 0248801

[17] Ito, T.: On finite Thurston-type orderings of braid groups. Groups Complex. Cryptol. 2, 123 155 (2010) Zbl pre05873470 MR 2747146

[18] Larue, D. M.: Left-distributive and left-distributive idempotent algebras. Ph.D. thesis, Univ. of Colorado, Boulder (1994) MR 2692162 